\title{
Skewed Generalized Error Distribution of Financial Assets and Option Pricing
}

\author{
Panayiotis Theodossiou* \\ Cyprus University of Technology, Cyprus
}

This article provides a mathematical and empirical investigation of the reasons for the presence of skewness and kurtosis in financial data. The results indicate that this phenomenon is triggered by higher-order moment dependencies in the data, such as asymmetric and conditional volatility. Moreover, the article develops and tests successfully a skewed extension of the generalized error distribution (SGED), which is then used to model European call option prices. Under the standard assumptions of risk neutrality, normality of log-returns, and absence of arbitrage opportunities, the SGED model yields as special cases several well-known models for pricing options on stocks, stock indices, currencies, and currency futures. (JEL: C13, C22, G12, G13)

Keywords: asymmetric volatility; call option pricing; conditional heteroskedasticity; geometric Brownian motion; skewed GED

\section{Introduction}

Commonly used option pricing models, including that of Black-Scholes, are based on the assumption that prices of financial assets follow the geometric Brownian motion. A key assumption of the geometric Brownian motion is that the ratio of two consecutive prices does not

\footnotetext{
* Department of Shipping and Finance, Cyprus University of Technology, Cyprus. Tel: +357 25002340, e-mail: theodoss@gmail.com. The author would like to thank George Constantinides, Christodoulos Louca, Christos Savva, Dick Stapleton, Lenos Trigeorgis, Jack Worrall and Uzi Yaari for helpful comments. This article benefitted from presentations at the School of Engineering Management of the University of Chania, Greece, the University of Cyprus and the annual conferences of the Multinational Finance Society and the European Financial Management Association. An earlier version of the paper was circulated under the title "Distribution of Financial Asset Prices, the Skewed Generalized Error Distribution and the Pricing of Options."
}

(Multinational Finance Journal, 2015, vol. 19, no. 4, pp. 223-266)

(C) Multinational Finance Society, a nonprofit corporation. All rights reserved. DOI: $10.17578 / 19-4-1$ 
depend on past prices. Furthermore, the natural logarithm of two consecutive prices, known as the log-return, is assumed to be normally distributed with the same annualized mean and variance over time. ${ }^{1}$

The geometric Brownian motion's assumptions imply that log-returns are identically and independently distributed (i.i.d.) normal variables, thus they exhibit no moment dependencies, such as asymmetric and conditional volatility. The presence of conditional volatility in the log-return series of financial assets is well documented in the GARCH literature, e.g., Akgiray (1989) and Ballie and Bollerslev (1989). The EGARCH literature further documents that volatility is larger during market downturns than market upturns (asymmetric volatility), e.g., Hsieh (1989), Nelson (1991), and Booth, Martikainen, and Tse (1997). As shown in this article, these dependencies are likely to trigger skewness and kurtosis in of log-return distributions.

The nature of option pricing necessitates the use of probability distributions which provide a good fit to the empirical distribution of log-returns accommodating skewness and kurtosis. These distributions are required to have all their moments defined, i.e., have a moment generating function and include the normal distribution as a special case. The latter assumption is consistent with the assumption that price changes follow a geometric Brownian motion.

This article provides a mathematical and empirical investigation of the reasons for the presence of skewness and kurtosis in financial data. To accommodate these properties, the article formally develops a skewed extension of the generalized error distribution (SGED) and tests its ability to model the empirical distribution of log-returns of several popular financial series. The SGED is then used to derive a pricing model for European call options that accounts for skewness and kurtosis. As shown in the article, the SGED model nests several well-known call option pricing models for stocks, stock indices, currencies, and currency futures.

The article is organized as follows. The next section presents a preliminary statistical analysis of the data. Section III investigates the reasons for the presence of skewness and kurtosis in the data. Section IV discusses the implications of skewness and kurtosis for the geometric

1. The log-return is $y_{t}=\ln \left(P_{t} / P_{t-1}\right)=\ln \left(1+r_{t}\right)$, where $r_{t}$ is the return for the period $t-1$ to $t$. The return $r_{t}$ could be expressed in terms of $y_{t}$, as $r_{t}=\exp \left(y_{t}\right)-1$. Unlike returns, log-returns can be added across periods. For example, a monthly log-return is equal to the sum of daily $\log$-returns during the month, i.e., $Y_{n}=\Sigma y_{t}$, where $n$ is the number daily log-returns. The linear nature of log-returns simplifies considerably the computation of annualized means and variances of log-returns. 
TABLE 1. Financial Series

\begin{tabular}{lllrc}
\hline No & Name & Symbol & OBS & Period of Data \\
\hline 1. & Boeing & BA & 10,091 & $1 / 3 / 1975-12 / 31 / 2014$ \\
2. & IBM & IBM & 10,091 & $1 / 3 / 1975-12 / 31 / 2014$ \\
3. & DJIA & \$INDU & 10,435 & $1 / 2 / 1975-12 / 31 / 2014$ \\
4. & S\&P100 & \$OEX & 8,042 & $3 / 6 / 1984-12 / 31 / 2014$ \\
5. & S\&P500 & \$INX & 10,435 & $1 / 2 / 1975-12 / 31 / 2014$ \\
6. & GBP/USD & GBP/USD & 10,435 & $1 / 2 / 1975-12 / 31 / 2014$ \\
7. & JY/USD & JY/USD & 7,564 & $1 / 3 / 1986-12 / 31 / 2014$
\end{tabular}

Note: The stocks and currencies included in the above list are actively traded in U.S. financial markets. Options, futures, and other derivative assets written on them are very popular in the U.S. The currencies are expressed in terms of the U.S. dollar.

Brownian motion. Section V derives and applies the SGED to the data. Section VI presents the derivations for the SGED option pricing model. The last section offers the conclusions.

\section{Data and Preliminary Findings}

Table 1 lists the financial series and periods covered by the data. The data include daily, weekly, monthly and quarterly log-returns for two widely-held stocks (Boeing and IBM), three stock indices (Dow Jones Industrial Average (DJIA), S\&P100, and S\&P500), and two U.S. dollar exchange rates (British pound and Japanese yen). All of these assets have actively traded options. For example, call options on Boeing and IBM stocks and on the DJIA, S\&P100, and S\&P500 stock indices are actively traded on the Chicago Board of Options Exchange (CBOE). The data spans the period January 2, 1975 to December 31, 2014.

Log-returns at all frequencies are computed using the formula

$$
y_{t}=100 \times \ln \left(P_{t} / P_{t-1}\right) \text {, }
$$

where $P_{t}$ is the price at the end of period $t$. For example, using monthly data, $P_{t-1}$ and $P_{t}$ and are the closing prices on the last trading day of two consecutive months $t-1$ and $t$. It can be readily shown that the additive property implies that a monthly (or quarterly) log-return is equal to the sum of all daily returns during the month (or quarter). As a consequence, statistics such as the mean and variance of lower 
frequency data can be derived from those of higher frequency data.

Table 2 reports basic statistics of log-returns for all series examined, starting with the mean, the standard deviation, and the minimum and maximum values of log-returns in the first four rows. To allow comparison across the four data frequencies, the statistics are annualized using the formulas $\bar{y} / \Delta t, S / \sqrt{\Delta t}, \min / \Delta t$, and max $/ \Delta t$, where $\Delta t=1 / 252,1 / 52,1 / 12$ and $1 / 4$, for the daily, weekly, monthly and quarterly frequencies, respectively, where $\bar{y}$ is the simple arithmetic mean and $S$ the sample standard deviation.

The annualized means for the stocks and stock indices range between $4.6552 \%$ and $14.0506 \%$ and the means for the two currencies between $-0.9355 \%$ and $-2.0902 \%$. Note that the mean and standard deviation of log-returns for each series are quite similar across the four data frequencies examined; a result which is due to the additive property of log-returns and the linearity of the mean estimator. The similarity of standard deviations across the four data frequencies suggests that data of any frequency may be used to estimate the mean and volatility of asset log-price changes. In most cases, the standard deviations of logreturns are larger than their means, indicating high volatility. This phenomenon is confirmed by the large discrepancies between the minimum and maximum values of log-returns.

The next four rows give the statistics and t-values for standardized skewness and standardized kurtosis in excess of three, which is the standardized kurtosis for the normal distribution, computed using the equations

$$
b_{1}=\hat{m}_{3} / \hat{m}_{2}^{3 / 2}
$$

and

$$
b_{2}=\left(\hat{m}_{4} / \hat{m}_{2}^{2}\right)-3
$$

where

$$
\hat{m}_{j}=\sum\left(y_{t}-\bar{y}\right)^{j} / T
$$

is the estimate for the $j^{\text {th }}$ moment around the sample mean. Under the null hypothesis of normality, the two statistics are normally distributed with standard errors $S E\left(b_{1}\right)=\sqrt{6 / T}$ and $S E\left(b_{1}\right)=\sqrt{6 / T}$.

In general, the data series exhibit significant negative skewness across all four frequencies. The only exceptions are the daily and weekly log-returns for Boeing and the monthly log-returns for IBM. The excess kurtosis values are statistically significant in all cases implying 
TABLE 2. Preliminary Statistics

\begin{tabular}{|c|c|c|c|c|}
\hline Estimates & Daily & Weekly & Monthly & Quarterly \\
\hline \multicolumn{5}{|l|}{ A. Boeing } \\
\hline Mean & 13.9601 & 13.9724 & 14.0506 & 13.9853 \\
\hline Standard Deviation & 31.6945 & 31.7474 & 31.3298 & 32.6269 \\
\hline minimum & $-4,886.1057$ & $-1,227.4442$ & -519.4369 & -253.0499 \\
\hline maximum & $8,903.5831$ & $2,035.2159$ & 406.4927 & 186.1446 \\
\hline \multirow{2}{*}{$b_{1}$} & 0.415 & 0.1017 & -0.2593 & -0.3717 \\
\hline & $(17.02)^{* *}$ & $(1.9)$ & $(-2.32)^{*}$ & $(-1.91)$ \\
\hline \multirow[t]{2}{*}{$b_{2}$} & 14.0881 & 4.7511 & 2.1454 & 1.2939 \\
\hline & $(288.88)^{* *}$ & $(44.3)^{* *}$ & $(9.58)^{* *}$ & $(3.33)^{* *}$ \\
\hline$B J$ & $83,739.36^{* *}$ & $1,966.5^{* *}$ & $97.23 * *$ & $14.75 * *$ \\
\hline Observations & 10,091 & 2,087 & 479 & 159 \\
\hline \multicolumn{5}{|l|}{ B. IBM } \\
\hline Mean & 4.6647 & 4.6552 & 4.6967 & 5.1069 \\
\hline Standard Deviation & 26.5701 & 27.2054 & 24.9627 & 26.0154 \\
\hline minimum & $-6,574.2738$ & $-1,887.1086$ & -360.7416 & -187.3526 \\
\hline maximum & $3,116.3503$ & $1,196.0854$ & 355.4328 & 153.1533 \\
\hline \multirow[t]{2}{*}{$b_{1}$} & -0.3289 & -0.3819 & 0.089 & -0.2594 \\
\hline & $(-13.49)^{* *}$ & $(-7.12)^{* *}$ & $(0.8)$ & $(-1.34)$ \\
\hline \multirow[t]{2}{*}{$b_{2}$} & 12.9657 & 7.8463 & 1.6506 & 1.4358 \\
\hline & $(265.86)^{* *}$ & $(73.17)^{* *}$ & $(7.37)^{* *}$ & $(3.7)^{* *}$ \\
\hline$B J$ & $70,865.24 * *$ & $5,404.25^{* *}$ & $55.01 * *$ & $15.44 * *$ \\
\hline Observations & 10,091 & 2,087 & 479 & 159 \\
\hline \multicolumn{5}{|l|}{ C. DJIA } \\
\hline Mean & 8.4156 & 8.4132 & 8.4226 & 8.3164 \\
\hline Standard Deviation & 17.1063 & 17.6489 & 15.4373 & 16.1252 \\
\hline minimum & $-6,689.9406$ & $-1,828.5294$ & -324.37 & -123.3565 \\
\hline maximum & $2,742.6787$ & 681.0413 & 172.3855 & 84.7035 \\
\hline \multirow[t]{2}{*}{$b_{1}$} & -1.4536 & -1.4609 & -0.7128 & -0.7582 \\
\hline & $(-60.62)^{* *}$ & $(-27.25)^{* *}$ & $(-6.37)^{* *}$ & $(-3.9)^{* *}$ \\
\hline \multirow[t]{2}{*}{$b_{2}$} & 40.2445 & 22.9967 & 3.7743 & 1.4467 \\
\hline & $(839.16)^{* *}$ & $(214.45)^{* *}$ & $(16.86)^{* *}$ & $(3.72)^{* *}$ \\
\hline$B J$ & $707,872.46^{* *}$ & $46,729.92 * *$ & $324.88 * *$ & $29.1 * *$ \\
\hline Observations & 10,435 & 2,087 & 479 & 159 \\
\hline \multicolumn{5}{|l|}{ D. S\&P100 } \\
\hline Mean & 7.9908 & 8.0110 & 8.0155 & 7.8642 \\
\hline Standard Deviation & 18.69 & 18.4228 & 16.008 & 16.8535 \\
\hline minimum & $-6,183.0804$ & $-1,743.8139$ & -289.2516 & -118.9073 \\
\hline maximum & $2,780.9704$ & 621.661 & 168.4502 & 92.9781 \\
\hline
\end{tabular}

( Continued) 
TABLE 2. (Continued)

\begin{tabular}{|c|c|c|c|c|}
\hline Estimates & Daily & Weekly & Monthly & Quarterly \\
\hline \multicolumn{5}{|l|}{ D. S\&P100 } \\
\hline \multirow[t]{2}{*}{$b_{1}$} & -1.2466 & -1.5973 & -0.9063 & -0.9047 \\
\hline & $(-45.64)^{* *}$ & $(-26.15)^{* *}$ & $(-7.11)^{* *}$ & $(-4.1)^{* *}$ \\
\hline \multirow[t]{2}{*}{$b_{2}$} & 29.4299 & 21.1519 & 3.0421 & 1.7858 \\
\hline & $(538.72)^{* *}$ & $(173.14)^{* *}$ & $(11.93)^{* *}$ & $(4.04)^{* *}$ \\
\hline BJ & $292,305.48 * *$ & $30,659.75^{* *}$ & $192.8^{* *}$ & $33.12 * *$ \\
\hline Observations & 8,042 & 1,608 & 369 & 123 \\
\hline \multicolumn{5}{|l|}{ E. S\&P500 } \\
\hline Mean & 8.5096 & 8.5151 & 8.5166 & 8.4174 \\
\hline Standard Deviation & 17.3256 & 17.7345 & 15.4574 & 16.3197 \\
\hline minimum & $-5,976.8276$ & $-1,659.9106$ & -296.1221 & -112.5027 \\
\hline maximum & $2,859.8281$ & 673.4488 & 175.3387 & 88.0366 \\
\hline \multirow[t]{2}{*}{$b_{1}$} & -1.1473 & -1.2177 & -0.8325 & -0.8071 \\
\hline & $(-47.85)^{* *}$ & $(-22.71)^{* *}$ & $(-7.44)^{* *}$ & $(-4.15)^{* *}$ \\
\hline \multirow[t]{2}{*}{$b_{2}$} & 28.2369 & 16.7235 & 3.3502 & 1.331 \\
\hline & $(588.79)^{* *}$ & $(155.95)^{* *}$ & $(14.97)^{* *}$ & $(3.43)^{* *}$ \\
\hline BJ & $348,957.75^{* *}$ & $24,835.95^{* *}$ & $279.33 * *$ & $29 * *$ \\
\hline Observations & 10,435 & 2,087 & 479 & 159 \\
\hline \multicolumn{5}{|l|}{ F. GBP/USD } \\
\hline Mean & -1.026 & -1.0322 & -1.0069 & -0.9355 \\
\hline Standard Deviation & 9.6346 & 10.2107 & 10.4678 & 10.5994 \\
\hline minimum & $-1,022.6542$ & -530.2511 & -167.7294 & -83.273 \\
\hline maximum & $1,218.1852$ & 330.4369 & 157.0867 & 59.9604 \\
\hline \multirow[t]{2}{*}{$b_{1}$} & -0.0528 & -0.469 & -0.3306 & -0.438 \\
\hline & $(-2.2)^{*}$ & $(-8.75)^{* *}$ & $(-2.95)^{* *}$ & $(-2.25)^{*}$ \\
\hline \multirow[t]{2}{*}{$b_{2}$} & 4.0676 & 3.8873 & 2.097 & 1.3898 \\
\hline & $(84.82)^{* *}$ & $(36.25)^{* *}$ & $(9.37)^{* *}$ & $(3.58)^{* *}$ \\
\hline$B J$ & $7,198.56^{* *}$ & $1,390.54 * *$ & $96.49 * *$ & $17.88 * *$ \\
\hline Observations & 10,435 & 2,087 & 479 & 159 \\
\hline \multicolumn{5}{|l|}{ G. JY/USD } \\
\hline Mean & -1.7475 & -1.7123 & -1.7891 & -2.0902 \\
\hline Standard Deviation & 11.3237 & 11.5016 & 11.4819 & 12.4587 \\
\hline minimum & $-2,003.7751$ & -691.8273 & -199.5999 & -75.3561 \\
\hline maximum & $1,521.7646$ & 328.4774 & 122.2159 & 67.7685 \\
\hline \multirow[t]{2}{*}{$b_{1}$} & -0.4264 & -0.6155 & -0.342 & -0.4069 \\
\hline & $(-15.14)^{* *}$ & $(-9.77)^{* *}$ & $(-2.6)^{* *}$ & $(-1.78)$ \\
\hline \multirow[t]{2}{*}{$b_{2}$} & 6.9059 & 4.5649 & 1.6913 & 0.426 \\
\hline & $(122.6)^{* *}$ & $(36.24)^{* *}$ & $(6.43)^{* *}$ & $(0.93)$ \\
\hline$B J$ & $15,260.11^{* *}$ & $1,409.18 * *$ & $48.13 * *$ & 4.04 \\
\hline Observations & 7,564 & 1,513 & 347 & 115 \\
\hline
\end{tabular}

( Continued) 
TABLE 2. (Continued)

Note: Log-returns for the period $t$ to $t-1$ are computed using $y_{t}=100 \times \ln \left(P_{t} / P_{t-1}\right)$, where $P_{t}$ is the price of the stock or the value of the currency or index at time $t$. The mean, variance, minimum and maximum return are based on annualized returns computed using $y_{t} / \Delta t$, where $\Delta t=1 / 252,1 / 52,1 / 12$, and $1 / 4$ for the daily, weekly, monthly and quarterly frequencies. The statistics for skewness and excess kurtosis are computed using $b_{1}=m_{3} / m_{2}{ }^{3 / 2}, b_{2}=\left(m_{4} / m_{2}{ }^{2}\right)$ -3 , where $m_{i}$ is the sample the $i^{\text {th }}$ centered moment. Parentheses include t-values statistics. The Bera-Jarque statistic for testing normality, $B J=(T / 24)\left(4 b_{1}{ }^{2}+b_{2}{ }^{2}\right)$, is distributed as $\chi^{2}(2)$ with 2 degrees of freedom.Its critical value at the five-percent level is 5.99 and a the onepercent level is $9.21{ }^{*},{ }^{* *}$ statistically significant at the five- and one-percent level of significance.

that the log-return are leptokurtic relative to the normal distribution.

The ninth row of table reports the Bera-Jarque statistics for testing the null hypothesis of normality, given by

$$
B J=(T / 24)\left(4 b_{1}^{2}+b_{2}^{2}\right)
$$

The $B J$ is asymptotically $\chi^{2}(2)$ with two degrees of freedom. Its critical values are 5.99 at the five-percent level and 9.21 at the one-percent level. The $B J$ statistics confirm the significance of skewness and/or leptokurtosis by rejecting the normality assumption in all series.

In summary, these preliminary results indicate that distributions of the log-return series are leptokurtosis and, in most cases, skewed. These findings are, however, based on the null hypothesis of normality and provide no information as to the parametric distribution of the data. Moreover, the statistics for skewness and kurtosis may be magnified by the presence of outliers.

\section{Causes of Skewness and Kurtosis}

The central limit theorem states that the distribution of the sum of i.i.d. random variables approaches the normal distribution as the number of variables summed-up increases. Provided that daily log-returns are i.i.d. random variables, the distributions of quarterly log-returns, each being the sum of about 63 non-overlapping daily log-returns, should be normal. However, this is not the case because of the fact that daily logreturns exhibit strong higher order moment dependencies such as, asymmetric volatility, conditional heteroskedasticity (volatility), or other dependencies. 
Let $Z_{n}=z_{1}+z_{2}+\ldots+z_{n}$ be the sum of $n$ non-overlapping random variables, where $z_{t}=\left(y_{t}-\mu\right) / \sigma$, and $\mu$ and $\sigma$ are the mean and standard deviation of the log-return $y_{t}$. As such, the third and fourth moments of $z_{t}$, denoted by $m_{3}$ and $m_{4}$, are the standardized skewness and kurtosis of $y_{t}$. The expected value of $Z_{n}$ is zero, i.e., $E\left(Z_{n}\right)=0$. Moreover, under the assumption of i.i.d. log-returns $E\left(z_{t} z_{s}\right)=0$, for $t \neq s$ and $\operatorname{var}\left(Z_{n}\right)=n$.

The third and fourth centered moments of $Z_{n}$ are

$$
\begin{gathered}
M_{3}=E Z_{n}^{3}=E\left(z_{1}+z_{2}+\cdots+z_{n}\right)^{3} \\
=\sum_{t} E z_{t}^{3}+3 \sum_{t \neq s} \sum E z_{t}^{2} z_{s}+\sum \sum_{t \neq s \neq p} \sum E z_{t} z_{s} z_{p}
\end{gathered}
$$

and

$$
\begin{gathered}
M_{4}=E Z_{n}^{4}=E\left(z_{1}+z_{2}+\cdots+z_{n}\right)^{4} \\
=\sum_{t} E z_{t}^{4}+4 \sum_{t \neq s} E z_{t}^{3} z_{s}+3 \sum_{t \neq s} E z_{t}^{2} z_{s}^{2} \\
+3 \sum_{t \neq s \neq p} \sum E z_{t}^{2} z_{s} z_{p}+\sum \sum_{t \neq s \neq p \neq r} \sum \sum E z_{t} z_{s} z_{p} z_{r} .
\end{gathered}
$$

Provided that $z_{t}$ are i.i.d., for $t \neq s \neq p \neq r$,

$$
\begin{gathered}
E z_{t} z_{s} z_{p}=0, \\
E z_{t}^{2} z_{s}=0, \\
E z_{t} z_{s} z_{p} z_{r}=0, \\
E z_{t}^{2} z_{s} z_{p}=0, \\
E z_{t}^{2} z_{s}^{2}=E z_{t}^{2} E z_{s}^{2}=1,
\end{gathered}
$$

and

$$
E z_{t}^{3} z_{s}=0 .
$$


Deviations of the above measures from their null values are evidence of the existence of higher order moment dependencies in the distribution of log-returns. Their substitutions into equations (6) and (7) give

and

$$
M_{3}=E Z_{n}^{3}=n m_{3}
$$

$$
M_{4}=E Z_{k}^{4}=n m_{4}+3 n(n-1) .
$$

where $m_{3}=E z^{3}$ and $m_{4}=E z^{4}$ are respectively the standardized skewness and standardized kurtosis measures for $z$ and $y$ as well. The skewness and kurtosis for $Z_{n}$ are

$$
S K=\frac{E Z_{n}^{3}}{\operatorname{var}\left(Z_{n}\right)^{3 / 2}}=\frac{n m_{3}}{n^{3 / 2}}=\frac{m_{3}}{\sqrt{n}}
$$

and

$$
K U=\frac{E Z_{n}^{4}}{\operatorname{var}\left(Z_{n}\right)^{2}}=\frac{n m_{4}+3 n(n-1)}{n^{2}}=\frac{m_{4}}{n}+3\left(1-\frac{1}{n}\right)
$$

It follows easily from the above equations that as $n \rightarrow \infty$ the skewness measure $S K$ approaches the value of zero and the kurtosis $K U$ the value of three which the standardized skewness and kurtosis values for the normal distribution.

Equations (6) and (7) provide the foundation for constructing test statistics for higher-order moment dependencies in financial data,

$$
\begin{gathered}
m z_{1}=\frac{\sum \hat{z}_{t}^{2} \hat{z}_{t-j} \text { and } \operatorname{var}\left(m z_{1}\right)=\frac{E z_{t}^{4}}{T}}{T} \\
m z_{2}=\frac{\sum \hat{z}_{t} \hat{z}_{t-1} \hat{z}_{t-2}}{T} \text { and } \operatorname{var}\left(m z_{2}\right)=\frac{1}{T}, \\
m z_{3}=\frac{\sum \hat{z}_{t}^{3} \hat{z}_{t-1}}{T} \text { and } \operatorname{var}\left(m z_{3}\right)=\frac{E z_{t}^{6}}{T}
\end{gathered}
$$




$$
\begin{gathered}
m z_{4}=\frac{\sum\left(\hat{z}_{t}^{2} \hat{z}_{t-j}^{2}-1\right)}{T} \text { and } \operatorname{var}\left(m z_{4}\right)=\frac{\left(E z_{t}^{4}\right)^{2}}{T}, \\
m z_{5}=\frac{\sum \hat{z}_{t}^{2} \hat{z}_{t-1} \hat{z}_{t-2}}{T} \text { and } \operatorname{var}\left(m z_{5}\right)=\frac{E z_{t}^{4}}{T}
\end{gathered}
$$

and

$$
m z_{6}=\frac{\sum \hat{z}_{t} \hat{z}_{t-1} \hat{z}_{t-2} \hat{z}_{t-3}}{T} \text { and } \operatorname{var}\left(m z_{6}\right)=\frac{1}{T}
$$

where $\hat{z}_{t}=\left(y_{t}-\bar{y}\right) / S, \bar{y}$ and $S$ are respectively the sample mean and standard deviation of $y_{t}$, and $T$ is the sample size and $j=1,2$ and 3. The moments $E z_{t}^{4}$ and $E z_{t}^{6}$ are estimated using respectively the statistics

$$
\hat{m}_{4}=\sum \hat{z}_{t}^{4} / T
$$

and

$$
\hat{m}_{6}=\sum \hat{z}_{t}^{6} / T
$$

The variances of the statistics above are derived under the null hypothesis that the sequence $z_{t}$ includes i.i.d. random variables, which implies that all the statistics in (18) have a zero mean. Note that there is a large number of statistics generated by (6) and (7) Thus, the above test statistics are not exhaustive of all higher-order moment dependencies in the data.

Table 3 presents the statistics given by (18a-f) along with their $t$ values for all log-return series and frequencies. Statistically significant monthly and occasionally in quarterly log-returns of all seven series. These relationships weaken with aggregation. Many of the dependencies documented on table 3 are consistent with earlier results on the stochastic nature of log-returns of financial assets. This literature reports that volatility is significantly clustered (conditional heteroskedasticity), or that large log-returns are likely to be followed by large log-returns of lesser magnitude, but undetermined sign. The latter implies

$$
E z_{t}^{2} z_{s}^{2} \neq E z_{t}^{2} E z_{s}^{2}, \text { for } t \neq s
$$

The statistics based on $z_{t}^{2} z_{s}^{2}$ for $s=t-1, t-2$ and $t-3$, test for conditional heteroskedasticity in the data, the presence of which is revealed in all 
TABLE 3. Higher Order Moment Dependencies

\begin{tabular}{|c|c|c|c|c|}
\hline Estimates & Daily & Weekly & Monthly & Quarterly \\
\hline \multicolumn{5}{|l|}{ A. Boeing } \\
\hline$z_{t}^{2} z_{t-1}$ & $\begin{array}{l}-0.0965 \\
(-2.35)^{*}\end{array}$ & $\begin{array}{l}-0.21 \\
(-3.45)^{* *}\end{array}$ & $\begin{array}{l}-0.172 \\
(-1.66)\end{array}$ & $\begin{array}{c}0.0307 \\
(0.19)\end{array}$ \\
\hline$z_{t}^{2} z_{t-2}$ & $\begin{array}{c}-0.1377 \\
(-3.35)^{* *}\end{array}$ & $\begin{array}{l}-0.1126 \\
(-1.85)\end{array}$ & $\begin{array}{l}0.0999 \\
(0.96)\end{array}$ & $\begin{array}{l}-0.3844 \\
(-2.35)^{*}\end{array}$ \\
\hline$z_{t}^{2} z_{t-3}$ & $\begin{array}{c}-0.1573 \\
(-3.82)^{* *}\end{array}$ & $\begin{array}{l}-0.0943 \\
(-1.55)\end{array}$ & $\begin{array}{c}-0.2778 \\
(-2.68)^{* *}\end{array}$ & $\begin{array}{l}-0.308 \\
(-1.88)\end{array}$ \\
\hline$z_{t} z_{t-1} z_{t-2}$ & $\begin{array}{l}-0.0288 \\
(-0)\end{array}$ & $\begin{array}{l}0.0653 \\
(0)\end{array}$ & $\begin{array}{l}0.0562 \\
(0)\end{array}$ & $\begin{array}{l}-0.0879 \\
(-0.01)\end{array}$ \\
\hline$z_{t}^{3} z_{t-1}$ & $\begin{array}{c}0.0031 \\
(0.01)\end{array}$ & $\begin{array}{l}0.0054 \\
(0.01)\end{array}$ & $\begin{array}{c}1.1194 \\
(3.14)^{* *}\end{array}$ & $\begin{array}{l}0.5817 \\
(1.16)\end{array}$ \\
\hline$z_{t}^{2} z_{t-1}^{2}-1$ & $\begin{array}{c}1.1287 \\
(6.64)^{* *}\end{array}$ & $\begin{array}{c}0.5898 \\
(3.48)^{* *}\end{array}$ & $\begin{array}{l}0.4148 \\
(1.77)\end{array}$ & $\begin{array}{l}-0.0166 \\
(-0.05)\end{array}$ \\
\hline$z_{t}^{2} z_{t-2}^{2}-1$ & $\begin{array}{c}0.725 \\
(4.26)^{* *}\end{array}$ & $\begin{array}{c}0.4735 \\
(2.79)^{* *}\end{array}$ & $\begin{array}{c}0.3771 \\
(1.61)\end{array}$ & $\begin{array}{l}0.1155 \\
(0.34)\end{array}$ \\
\hline$z_{t}^{2} z_{t-3}^{2}-1$ & $\begin{array}{c}0.806 \\
(4.74)^{* *}\end{array}$ & $\begin{array}{l}0.2809 \\
(1.66)\end{array}$ & $\begin{array}{c}0.8353 \\
(3.56)^{* *}\end{array}$ & $\begin{array}{l}0.0174 \\
(0.05)\end{array}$ \\
\hline$z_{t}^{2} z_{t-1} z_{t-2}$ & $\begin{array}{c}0.1832 \\
(4.45)^{* *}\end{array}$ & $\begin{array}{l}0.0646 \\
(1.06)\end{array}$ & $\begin{array}{l}-0.0966 \\
(-0.93)\end{array}$ & $\begin{array}{l}0.2156 \\
(1.32)\end{array}$ \\
\hline$z_{t} z_{t-1} z_{t-2} z_{t-3}$ & $\begin{array}{c}0.0523 \\
(5.26)^{* *}\end{array}$ & $\begin{array}{l}-0.0206 \\
(-0.94)\end{array}$ & $\begin{array}{l}-0.1298 \\
(-2.84)^{* *}\end{array}$ & $\begin{array}{l}0.1138 \\
(1.43)\end{array}$ \\
\hline \multicolumn{5}{|l|}{ B. IBM } \\
\hline$z_{t}^{2} z_{t-1}$ & $\begin{array}{l}-0.161 \\
(-4.05)^{* *}\end{array}$ & $\begin{array}{c}-0.2456 \\
(-3.41)^{* *}\end{array}$ & $\begin{array}{l}-0.2562 \\
(-2.6)^{* *}\end{array}$ & $\begin{array}{l}-0.3069 \\
(-1.84)\end{array}$ \\
\hline$z_{t}^{2} z_{t-2}$ & $\begin{array}{c}-0.1381 \\
(-3.47)^{* *}\end{array}$ & $\begin{array}{l}-0.0966 \\
(-1.34)\end{array}$ & $\begin{array}{l}-0.0944 \\
(-0.96)\end{array}$ & $\begin{array}{l}0.2382 \\
(1.43)\end{array}$ \\
\hline$z_{t}^{2} z_{t-3}$ & $\begin{array}{l}-0.0995 \\
(-2.5)^{*}\end{array}$ & $\begin{array}{l}-0.054 \\
(-0.75)\end{array}$ & $\begin{array}{l}-0.122 \\
(-1.24)\end{array}$ & $\begin{array}{l}-0.0712 \\
(-0.43)\end{array}$ \\
\hline$z_{t} z_{t-1} z_{t-2}$ & $\begin{array}{l}-0.0151 \\
(-0)\end{array}$ & $\begin{array}{l}0.0254 \\
(0)\end{array}$ & $\begin{array}{l}0.0592 \\
(0)\end{array}$ & $\begin{array}{c}0.1169 \\
(0.01)\end{array}$ \\
\hline$z_{t}^{3} z_{t-1}$ & $\begin{array}{r}0.461 \\
(1.08)\end{array}$ & $\begin{array}{l}0.2771 \\
(0.56)\end{array}$ & $\begin{array}{l}-0.2201 \\
(-0.72)\end{array}$ & $\begin{array}{l}0.5829 \\
(1.2)\end{array}$ \\
\hline$z_{t}^{2} z_{t-1}^{2}-1$ & $\begin{array}{c}1.9619 \\
(12.34)^{* *}\end{array}$ & $\begin{array}{c}0.7696 \\
(3.24)^{* *}\end{array}$ & $\begin{array}{l}0.4041 \\
(1.91)\end{array}$ & $\begin{array}{l}0.1625 \\
(0.46)\end{array}$ \\
\hline$z_{t}^{2} z_{t-2}^{2}-1$ & $\begin{array}{c}1.153 \\
(7.26)^{* *}\end{array}$ & $\begin{array}{c}0.7991 \\
(3.37)^{* *}\end{array}$ & $\begin{array}{l}0.3011 \\
(1.42)\end{array}$ & $\begin{array}{r}0.6947 \\
(1.99)^{*}\end{array}$ \\
\hline$z_{t}^{2} z_{t-3}^{2}-1$ & $\begin{array}{c}0.7974 \\
(5.02)^{* *}\end{array}$ & $\begin{array}{c}0.5243 \\
(2.21)^{*}\end{array}$ & $\begin{array}{c}0.5359 \\
(2.53)^{*}\end{array}$ & $\begin{array}{l}0.1366 \\
(0.39)\end{array}$ \\
\hline$z_{t}^{2} z_{t-1} z_{t-2}$ & $\begin{array}{c}0.089 \\
(2.24)^{*}\end{array}$ & $\begin{array}{c}-0.2333 \\
(-3.24)^{* *}\end{array}$ & $\begin{array}{l}0.0816 \\
(0.83)\end{array}$ & $\begin{array}{l}-0.4637 \\
(-2.78)^{* *}\end{array}$ \\
\hline$z_{t} z_{t-1} z_{t-2} z_{t-3}$ & $\begin{array}{c}0.0323 \\
(3.25)^{* *}\end{array}$ & $\begin{array}{c}0.0485 \\
(2.22)^{*}\end{array}$ & $\begin{array}{l}-0.0172 \\
(-0.38)\end{array}$ & $\begin{array}{c}-0.2355 \\
(-2.97)^{* *}\end{array}$ \\
\hline
\end{tabular}

( Continued) 
TABLE 3. (Continued)

\begin{tabular}{|c|c|c|c|c|}
\hline Estimates & Daily & Weekly & Monthly & Quarterly \\
\hline \multicolumn{5}{|l|}{ C. DJIA } \\
\hline$z_{t}^{2} z_{t-1}$ & $\begin{array}{c}-0.4917 \\
(-7.64)^{* *}\end{array}$ & $\begin{array}{c}-0.5419 \\
(-4.86)^{* *}\end{array}$ & $\begin{array}{l}-0.442 \\
(-3.72)^{* *}\end{array}$ & $\begin{array}{l}-0.0555 \\
(-0.33)\end{array}$ \\
\hline$z_{t}^{2} z_{t-2}$ & $\begin{array}{l}-0.5663 \\
(-8.8)^{* *}\end{array}$ & $\begin{array}{l}-0.2506 \\
(-2.25)^{*}\end{array}$ & $\begin{array}{l}-0.1045 \\
(-0.88)\end{array}$ & $\begin{array}{l}-0.0669 \\
(-0.4)\end{array}$ \\
\hline$z_{t}^{2} z_{t-3}$ & $\begin{array}{c}-0.3585 \\
(-5.57)^{* *}\end{array}$ & $\begin{array}{l}-0.148 \\
(-1.33)\end{array}$ & $\begin{array}{l}-0.026 \\
(-0.22)\end{array}$ & $\begin{array}{c}0.1786 \\
(1.07)\end{array}$ \\
\hline$z_{t} z_{t-1} z_{t-2}$ & $\begin{array}{l}-0.0423 \\
(-0)\end{array}$ & $\begin{array}{l}0.0463 \\
(0)\end{array}$ & $\begin{array}{l}0.1188 \\
(0.01)\end{array}$ & $\begin{array}{l}-0.1567 \\
(-0.01)\end{array}$ \\
\hline$z_{t}^{3} z_{t-1}$ & $\begin{array}{c}5.6172 \\
(4.07)^{* *}\end{array}$ & $\begin{array}{c}3.3654 \\
(2.32)^{*}\end{array}$ & $\begin{array}{l}0.445 \\
(0.8)\end{array}$ & $\begin{array}{l}-0.2178 \\
(-0.43)\end{array}$ \\
\hline$z_{t}^{2} z_{t-1}^{2}-1$ & $\begin{array}{c}4.4113 \\
(10.42)^{* *}\end{array}$ & $\begin{array}{c}1.9363 \\
(3.4)^{* *}\end{array}$ & $\begin{array}{c}0.8995 \\
(2.91)^{* *}\end{array}$ & $\begin{array}{c}0.144 \\
(0.41)\end{array}$ \\
\hline$z_{t}^{2} z_{t-2}^{2}-1$ & $\begin{array}{c}7.4108 \\
(17.51)^{* *}\end{array}$ & $\begin{array}{c}2.8301 \\
(4.98)^{* *}\end{array}$ & $\begin{array}{l}0.2878 \\
(0.93)\end{array}$ & $\begin{array}{l}0.0195 \\
(0.06)\end{array}$ \\
\hline$z_{t}^{2} z_{t-3}^{2}-1$ & $\begin{array}{c}3.6972 \\
(8.73)^{* *}\end{array}$ & $\begin{array}{c}1.4791 \\
(2.6)^{* *}\end{array}$ & $\begin{array}{l}0.4151 \\
(1.34)\end{array}$ & $\begin{array}{c}0.4481 \\
(1.28)\end{array}$ \\
\hline$z_{t}^{2} z_{t-1} z_{t-2}$ & $\begin{array}{l}0.0131 \\
(0.2)\end{array}$ & $\begin{array}{c}-0.5253 \\
(-4.71)^{* *}\end{array}$ & $\begin{array}{l}0.2275 \\
(1.91)\end{array}$ & $\begin{array}{l}0.1238 \\
(0.74)\end{array}$ \\
\hline$z_{t} z_{t-1} z_{t-2} z_{t-3}$ & $\begin{array}{c}0.9713 \\
(99.22)^{* *}\end{array}$ & $\begin{array}{c}0.1527 \\
(6.98)^{* *}\end{array}$ & $\begin{array}{l}-0.1127 \\
(-2.47)^{*}\end{array}$ & $\begin{array}{l}-0.0022 \\
(-0.03)\end{array}$ \\
\hline D. S\&P100 & & & & \\
\hline$z_{t}^{2} z_{t-1}$ & $\begin{array}{l}-0.5776 \\
(-9.1)^{* *}\end{array}$ & $\begin{array}{c}-0.5289 \\
(-4.32)^{* *}\end{array}$ & $\begin{array}{l}-0.618 \\
(-4.84)^{* *}\end{array}$ & $\begin{array}{l}-0.3163 \\
(-1.61)\end{array}$ \\
\hline$z_{t}^{2} z_{t-2}$ & $\begin{array}{c}-0.5553 \\
(-8.75)^{* *}\end{array}$ & $\begin{array}{c}-0.3253 \\
(-2.66)^{* *}\end{array}$ & $\begin{array}{l}-0.2132 \\
(-1.67)\end{array}$ & $\begin{array}{l}-0.1624 \\
(-0.83)\end{array}$ \\
\hline$z_{t}^{2} z_{t-3}$ & $\begin{array}{c}-0.3382 \\
(-5.33)^{* *}\end{array}$ & $\begin{array}{l}-0.202 \\
(-1.65)\end{array}$ & $\begin{array}{l}-0.1339 \\
(-1.05)\end{array}$ & $\begin{array}{l}0.1408 \\
(0.72)\end{array}$ \\
\hline$z_{t} z_{t-1} z_{t-2}$ & $\begin{array}{l}-0.0295 \\
(-0)\end{array}$ & $\begin{array}{l}0.0522 \\
(0)\end{array}$ & $\begin{array}{l}0.2118 \\
(0.01)\end{array}$ & $\begin{array}{l}-0.1613 \\
(-0.01)\end{array}$ \\
\hline$z_{t}^{3} z_{t-1}$ & $\begin{array}{c}4.2016 \\
(3.84)^{* *}\end{array}$ & $\begin{array}{c}2.9331 \\
(2.03)^{*}\end{array}$ & $\begin{array}{l}0.7676 \\
(1.52)\end{array}$ & $\begin{array}{l}-0.1474 \\
(-0.26)\end{array}$ \\
\hline$z_{t}^{2} z_{t-1}^{2}-1$ & $\begin{array}{c}5.4177 \\
(14.98)^{* *}\end{array}$ & $\begin{array}{c}2.0137 \\
(3.35)^{* *}\end{array}$ & $(5)^{* *}$ & $\begin{array}{c}0.2367 \\
(0.55)\end{array}$ \\
\hline$z_{t}^{2} z_{t-2}^{2}-1$ & $\begin{array}{c}5.8484 \\
(16.17)^{* *}\end{array}$ & $\begin{array}{c}3.0787 \\
(5.11)^{* *}\end{array}$ & $\begin{array}{c}0.6404 \\
(2.04)^{*}\end{array}$ & $\begin{array}{l}0.1138 \\
(0.27)\end{array}$ \\
\hline$z_{t}^{2} z_{t-3}^{2}-1$ & $\begin{array}{c}3.0715 \\
(8.49)^{* *}\end{array}$ & $\begin{array}{c}1.6822 \\
(2.79)^{* *}\end{array}$ & $\begin{array}{l}0.5724 \\
(1.82)\end{array}$ & $\begin{array}{l}0.7665 \\
(1.79)\end{array}$ \\
\hline$z_{t}^{2} z_{t-1} z_{t-2}$ & $\begin{array}{c}0.2717 \\
(4.28)^{* *}\end{array}$ & $\begin{array}{c}-0.6468 \\
(-5.28)^{* *}\end{array}$ & $\begin{array}{c}0.4206 \\
(3.29)^{* *}\end{array}$ & $\begin{array}{l}0.1216 \\
(0.62)\end{array}$ \\
\hline$z_{t} z_{t-1} z_{t-2} z_{t-3}$ & $\begin{array}{c}1.0070 \\
(90.3)^{* *}\end{array}$ & $\begin{array}{c}0.1776 \\
(7.12)^{* *}\end{array}$ & $\begin{array}{l}-0.252 \\
(-4.84)^{* *}\end{array}$ & $\begin{array}{l}-0.0646 \\
(-0.72)\end{array}$ \\
\hline
\end{tabular}

( Continued) 
TABLE 3. (Continued)

\begin{tabular}{|c|c|c|c|c|}
\hline Estimates & Daily & Weekly & Monthly & Quarterly \\
\hline \multicolumn{5}{|l|}{ E. S\&P500 } \\
\hline$z_{t}^{2} z_{t-1}$ & $\begin{array}{l}-0.5032 \\
(-9.2)^{* *}\end{array}$ & $\begin{array}{l}-0.501 \\
(-5.15)^{* *}\end{array}$ & $\begin{array}{l}-0.5956 \\
(-5.18)^{* *}\end{array}$ & $\begin{array}{l}-0.2369 \\
(-1.44)\end{array}$ \\
\hline$z_{t}^{2} z_{t-2}$ & $\begin{array}{l}-0.5066 \\
(-9.26)^{* *}\end{array}$ & $\begin{array}{l}-0.2919 \\
(-3)^{* *}\end{array}$ & $\begin{array}{l}-0.1784 \\
(-1.55)\end{array}$ & $\begin{array}{l}-0.1603 \\
(-0.97)\end{array}$ \\
\hline$z_{t}^{2} z_{t-3}$ & $\begin{array}{l}-0.3027 \\
(-5.53)^{* *}\end{array}$ & $\begin{array}{c}-0.2132 \\
(-2.19)^{*}\end{array}$ & $\begin{array}{l}-0.1563 \\
(-1.36)\end{array}$ & $\begin{array}{l}0.0799 \\
(0.49)\end{array}$ \\
\hline$z_{t} z_{t-1} z_{t-2}$ & $\begin{array}{l}0.0002 \\
(0)\end{array}$ & $\begin{array}{l}0.0604 \\
(0)\end{array}$ & $\begin{array}{l}0.1752 \\
(0.01)\end{array}$ & $\begin{array}{l}-0.1974 \\
(-0.02)\end{array}$ \\
\hline$z_{t}^{3} z_{t-1}$ & $\begin{array}{c}4.0284 \\
(4.18)^{* *}\end{array}$ & $\begin{array}{l}2.0241 \\
(1.86)\end{array}$ & $\begin{array}{c}0.9435 \\
(1.97)^{*}\end{array}$ & $\begin{array}{l}0.0972 \\
(0.21)\end{array}$ \\
\hline$z_{t}^{2} z_{t-1}^{2}-1$ & $\begin{array}{c}4.1478 \\
(13.57)^{* *}\end{array}$ & $\begin{array}{c}1.8058 \\
(4.18)^{* *}\end{array}$ & $\begin{array}{c}1.5873 \\
(5.48)^{* *}\end{array}$ & $\begin{array}{l}0.3118 \\
(0.91)\end{array}$ \\
\hline$z_{t}^{2} z_{t-2}^{2}-1$ & $\begin{array}{c}6.1953 \\
(20.26)^{* *}\end{array}$ & $\begin{array}{c}2.6703 \\
(6.19)^{* *}\end{array}$ & $\begin{array}{c}0.7719 \\
(2.67)^{* *}\end{array}$ & $\begin{array}{l}0.0942 \\
(0.28)\end{array}$ \\
\hline$z_{t}^{2} z_{t-3}^{2}-1$ & $\begin{array}{c}3.3222 \\
(10.87)^{* *}\end{array}$ & $\begin{array}{l}1.487 \\
(3.45)^{* *}\end{array}$ & $\begin{array}{c}0.732 \\
(2.53)^{*}\end{array}$ & $\begin{array}{l}0.4534 \\
(1.33)\end{array}$ \\
\hline$z_{t}^{2} z_{t-1} z_{t-2}$ & $\begin{array}{c}0.1349 \\
(2.46)^{*}\end{array}$ & $\begin{array}{l}-0.4591 \\
(-4.72)^{* *}\end{array}$ & $\begin{array}{c}0.5022 \\
(4.37)^{* *}\end{array}$ & $\begin{array}{c}0.23 \\
(1.4)\end{array}$ \\
\hline$z_{t} z_{t-1} z_{t-2} z_{t-3}$ & $\begin{array}{c}0.7692 \\
(78.57)^{* *}\end{array}$ & $\begin{array}{l}-0.0431 \\
(-1.97)^{*}\end{array}$ & $\begin{array}{c}-0.3686 \\
(-8.07)^{* *}\end{array}$ & $\begin{array}{l}-0.0313 \\
(-0.39)\end{array}$ \\
\hline \multicolumn{5}{|l|}{ F. GBP/USD } \\
\hline$z_{t}^{2} z_{t-1}$ & $\begin{array}{l}-0.1124 \\
(-4.32)^{* *}\end{array}$ & $\begin{array}{l}-0.0536 \\
(-0.93)\end{array}$ & $\begin{array}{l}-0.272 \\
(-2.64)^{* *}\end{array}$ & $\begin{array}{l}-0.3229 \\
(-1.95)\end{array}$ \\
\hline$z_{t}^{2} z_{t-2}$ & $\begin{array}{l}-0.0695 \\
(-2.67)^{* *}\end{array}$ & $\begin{array}{l}0.0481 \\
(0.84)\end{array}$ & $\begin{array}{l}-0.0254 \\
(-0.25)\end{array}$ & $\begin{array}{l}-0.0394 \\
(-0.24)\end{array}$ \\
\hline$z_{t}^{2} z_{t-3}$ & $\begin{array}{l}-0.0379 \\
(-1.46)\end{array}$ & $\begin{array}{l}-0.0504 \\
(-0.88)\end{array}$ & $\begin{array}{l}0.0235 \\
(0.23)\end{array}$ & $\begin{array}{l}-0.0337 \\
(-0.2)\end{array}$ \\
\hline$z_{t} z_{t-1} z_{t-2}$ & $\begin{array}{l}-0.0174 \\
(-0)\end{array}$ & $\begin{array}{l}-0.0403 \\
(-0)\end{array}$ & $\begin{array}{l}0.0337 \\
(0)\end{array}$ & $\begin{array}{l}0.0896 \\
(0.01)\end{array}$ \\
\hline$z_{t}^{3} z_{t-1}$ & $\begin{array}{c}0.8804 \\
(7.25)^{* *}\end{array}$ & $\begin{array}{c}0.733 \\
(2.57)^{*}\end{array}$ & $\begin{array}{l}0.5166 \\
(1.44)\end{array}$ & $\begin{array}{l}1.1708 \\
(2.41)^{*}\end{array}$ \\
\hline$z_{t}^{2} z_{t-1}^{2}-1$ & $\begin{array}{c}0.7569 \\
(10.94)^{* *}\end{array}$ & $\begin{array}{c}0.7717 \\
(5.12)^{* *}\end{array}$ & $\begin{array}{c}0.8117 \\
(3.49)^{* *}\end{array}$ & $\begin{array}{l}0.2926 \\
(0.85)\end{array}$ \\
\hline$z_{t}^{2} z_{t-2}^{2}-1$ & $\begin{array}{c}0.6582 \\
(9.51)^{* *}\end{array}$ & $\begin{array}{c}0.4755 \\
(3.16)^{* *}\end{array}$ & $\begin{array}{l}0.2927 \\
(1.26)\end{array}$ & $\begin{array}{l}0.6245 \\
(1.81)\end{array}$ \\
\hline$z_{t}^{2} z_{t-3}^{2}-1$ & $\begin{array}{c}0.7835 \\
(11.33)^{* *}\end{array}$ & $\begin{array}{c}0.9536 \\
(6.33)^{* *}\end{array}$ & $\begin{array}{l}0.1413 \\
(0.61)\end{array}$ & $\begin{array}{l}0.1748 \\
(0.51)\end{array}$ \\
\hline$z_{t}^{2} z_{t-1} z_{t-2}$ & $\begin{array}{c}0.2365 \\
(9.09)^{* *}\end{array}$ & $\begin{array}{l}-0.0423 \\
(-0.74)\end{array}$ & $\begin{array}{l}0.0554 \\
(0.54)\end{array}$ & $\begin{array}{l}-0.185 \\
(-1.12)\end{array}$ \\
\hline$z_{t} z_{t-1} z_{t-2} z_{t-3}$ & $\begin{array}{c}0.0404 \\
(4.13)^{* *}\end{array}$ & $\begin{array}{r}0.006 \\
(0.27)\end{array}$ & $\begin{array}{l}0.0296 \\
(0.65)\end{array}$ & $\begin{array}{l}-0.0298 \\
(-0.38)\end{array}$ \\
\hline
\end{tabular}

( Continued) 
TABLE 3. (Continued)

\begin{tabular}{lcccc}
\hline Estimates & Daily & Weekly & Monthly & Quarterly \\
\hline G. JY/USD & & & & \\
$z_{t}^{2} z_{t-1}$ & -0.2592 & -0.1378 & -0.0922 & -0.036 \\
$z_{t}^{2} z_{t-2}$ & $(-7.16)^{* *}$ & $(-1.95)$ & $(-0.79)$ & $(-0.21)$ \\
$z_{t}^{2} z_{t-3}$ & -0.1713 & -0.0025 & -0.2424 & -0.0011 \\
& $(-4.73)^{* *}$ & $(-0.03)$ & $(-2.09)^{*}$ & $(-0.01)$ \\
$z_{t} z_{t-1} z_{t-2}$ & -0.1118 & -0.0613 & 0.138 & -0.1684 \\
& $(-3.09)^{* *}$ & $(-0.87)$ & $(1.19)$ & $(-0.98)$ \\
$z_{t}^{3} z_{t-1}$ & 0.0416 & 0.0063 & 0.1156 & 0.0641 \\
$z_{t}^{2} z_{t-1}^{2}-1$ & $(0)$ & $(0)$ & $(0.01)$ & $(0.01)$ \\
& 0.2782 & -0.2931 & 0.039 & -0.0113 \\
$z_{t}^{2} z_{t-2}^{2}-1$ & $(1.14)$ & $(-0.69)$ & $(0.09)$ & $(-0.03)$ \\
& 1.2951 & 0.6842 & 0.1803 & -0.3811 \\
$z_{t}^{2} z_{t-3}^{2}-1$ & $(11.37)^{* *}$ & $(3.52)^{* *}$ & $(0.72)$ & $(-1.2)$ \\
$z_{t}^{2} z_{t-1} z_{t-2}$ & 0.8722 & 0.4493 & 0.3293 & 0.0697 \\
$z_{t} z_{t-1} z_{t-2} z_{t-3}$ & $(7.66)^{* *}$ & $(2.31)^{*}$ & $(1.31)$ & $(0.22)$ \\
Observations & 0.3597 & 0.3841 & 0.0544 & 0.0727 \\
& $(3.16)^{* *}$ & $(1.98)^{*}$ & $(0.22)$ & $(0.23)$ \\
& 0.1944 & -0.0373 & -0.0318 & 0.0103 \\
& $(5.37)^{* *}$ & $(-0.53)$ & $(-0.27)$ & $(0.06)$ \\
& 0.0406 & -0.0956 & -0.0083 & -0.0345 \\
& $(3.53)^{* *}$ & $(-3.72)^{* *}$ & $(-0.16)$ & $(-0.37)$ \\
& & 1,513 & 347 & 115 \\
\hline
\end{tabular}

Note: The random variable $z=(y-\mu) / \sigma$ is standardized, i.e., ita has mean ero and standard deviation one. The table presents the mean values of the constructs over the entire sampling period across the four data frequencies. The parameter $\mu$ and $\sigma$ are replaced by the simple arithmetic mean and sample standard deviation. Parentheses include the $t$-values for the estimates. *,** implies statistically significant higher order moment dependencies at the five- and one-percent level of significance.

daily and weekly log-returns. Similar relationships for stocks, stock indices, and exchange rates are documented by a number of authors including Akgiray (1989) and Baillie and Bollerslev (1989).

The EGARCH literature reports that stock market volatility is larger during market downturns than market upturns, e.g., Nelson (1991), and Booth, Martikainen, and Tse (1997), Chiang et al. (2013), Deleze and Houssain (2014), Ferguson et al (2015), Gulati et al. (2013). This finding implies that

$$
E\left(z_{t}^{2} \mid z_{s}<0\right)>E\left(z_{t}^{2} \mid z_{s}>0\right)
$$

or that

$$
E z_{t}^{2} z_{s} \neq 0, \text { for } t>s
$$


Statistics based on the product $z_{t}^{2} z_{s}$, for $s=t-1, t-2$ and $t-3$, test for the presence of asymmetric volatility in the data. The statistics reported in table 3 reveal the presence of asymmetric volatility in almost all daily, weekly and monthly log-return series. The negative statistics imply that volatility is higher in a bearish market than in a bullish one.

The remaining statistics test for more complex higher-order moment dependencies (or non-linearities) that may cause skewness and kurtosis in lower frequency data. The results document such dependencies at the daily, weekly and monthly data frequencies. These higher order-moment dependencies are responsible for skewness and kurtosis in financial logreturn data; see equations (6) and (7).

\section{Geometric Brownian Motion}

Various option pricing models, including that of Black-Scholes, are based on the assumption that prices of financial assets follow the geometric Brownian motion. As a result, the ratio of two consecutive prices does not depend on past prices and its log-value (log-returns) and is normally distributed. Therefore, the ratio of two consecutive prices is log-normally distributed. The mathematical relation between logreturns $\left(\Delta \ln P_{t}\right)$ and geometric returns $\left(\Delta P_{t} / P_{t}\right)$ is as follows:

$$
\Delta \ln P_{t}=\ln \left(\frac{P_{t+\Delta t}}{P_{t}}\right)=\ln \left(1+\frac{\Delta P_{t}}{P_{t}}\right)
$$

Let $\mu_{a} \Delta t$ and $\sigma_{a}^{2} \Delta t$ be the mean and variance of $\Delta \ln P_{t}$, where $\mu_{a}$ and $\sigma_{a}^{2}$ are annualized measures and $\Delta t$ is the length of time between two consecutive prices expressed as a fraction of the year, i.e., for daily logreturns $\Delta t=1 / 252$. Consider the transformation

$$
z \equiv \frac{\Delta \ln P_{t}-\mu_{a} \Delta t}{\sigma_{a} \sqrt{\Delta t}}
$$

The log-return can be written as

$$
\Delta \ln P_{t}=\mu_{a} \Delta t+\sigma_{a} \sqrt{\Delta t} z
$$

where $z$ is a standardized random variable. Under normality 


$$
\lim _{\Delta t \rightarrow \infty} \Delta \ln P_{t}=d \ln P_{t}=\mu_{a}+\sigma_{a} d z
$$

where $d z=z \sqrt{d t}$ is a Wiener process. Equation (22) can be rewritten in terms of geometric returns as

$$
1+\Delta P_{t} / P_{t}=\exp \left(\mu_{a} \Delta t+\sigma_{a} \sqrt{\Delta t} z\right)
$$

Its moment function is ${ }^{2}$

$$
E\left(1+\Delta P_{t} / P_{t}\right)^{r}=\exp \left(\left(r \mu_{a}+\frac{1}{2} r^{2} \sigma_{a}^{2}\right) \Delta t\right)
$$

It follows from the above that ${ }^{3}$

$$
E\left(\Delta P_{t} / P_{t}\right)=\exp \left(\left(\mu_{a}+\frac{1}{2} \sigma_{a}^{2}\right) \Delta t\right)-1 \approx\left(\mu_{a}+\frac{1}{2} \sigma_{a}^{2}\right) \Delta t
$$

and

$$
\begin{aligned}
& \operatorname{var}\left(\Delta P_{t} / P_{t}\right)=E\left(1+\Delta P_{t} / P_{t}\right)^{2}-\left(E\left(1+\Delta P_{t} / P_{t}\right)\right)^{2} \\
= & \exp \left(\left(2 \mu_{a}+2 \sigma_{a}^{2}\right) \Delta t\right)-\exp \left(\left(2 \mu_{a}+\sigma_{a}^{2}\right) \Delta t\right) \approx \sigma_{a}^{2} \Delta t .
\end{aligned}
$$

Along the same lines as with equation (22)

$$
\Delta P_{t} / P_{t}=\left(\mu_{a}+\frac{1}{2} \sigma_{a}^{2}\right) \Delta t+\sigma_{a} \sqrt{\Delta t} z
$$

where $z$ is a standardized log-normal random variable. As $\Delta t \rightarrow 0$, the above equation yields that of the geometric Brownian motion

$$
d P_{t}=\left(\mu_{a}+\frac{1}{2} \sigma_{a}^{2}\right) P_{t} d t+\sigma_{a} P_{t} d z
$$

where $\left(\mu_{a}+\frac{1}{2} \sigma_{a}^{2}\right) P_{t}$ and $\sigma_{a} P_{t}$ are the instantaneous drift the standard

2. The moment function of $x=e^{y}$, where $y$ is a normal with mean $\mu$ and variance $\sigma^{2}$, is obtained from the moment generating function of $y$, i.e., $E x^{r}=E e^{r y}=\exp \left(r \mu+\frac{1}{2} r^{2} \sigma^{2}\right)$.

3. $e^{x}=1+x+x^{2} / 2 !+x^{3} / 3 !+\cdots$, where $x=\left(r \mu_{a}+\frac{1}{2} r^{2} \sigma_{a}^{2}\right) \Delta t$. Note that the terms of the expansion of order two or greater vanish as the interval $\Delta t$ becomes short. 
deviation of the process.

Ross (1999) shows that the GBM could also be obtained as a limit of a simpler process. For example, consider the ratio of two consecutive prices $P_{n} / P_{0}$. The logarithm of the ratio

$$
Y_{n}=\ln \left(P_{n} / P_{0}\right)=\sum_{t=1}^{n} \ln \left(P_{t} / P_{t-1}\right)
$$

could be broken down into the sum of $n$ higher frequency log-returns corresponding to period $\Delta t$ and having mean $\mu_{a} \Delta t$ and variance $\sigma_{a}^{2} \Delta t$. Under the assumption of i.i.d. log-returns, the log-return $Y_{n}$ has a mean $E\left(Y_{n}\right)=\mu_{a} \tau$ and a variance $\operatorname{var}\left(Y_{n}\right)=\sigma_{a}^{2} \tau$, where $\tau=n \Delta t$ is the time between prices $P_{0}$ and $P_{n}$. For a large $n$, the central limit theorem states that $Y_{n}$ will be approximately normal, i.e.,

$$
P\left(\frac{Y_{n}-\mu_{a} \tau}{\sigma_{a} \sqrt{\tau}} \leq z\right) \approx \Phi(z),
$$

with the approximation becoming exact as $n \rightarrow \infty$.

The i.i.d. assumption for $P_{t} / P_{t-1}$ causes the $P_{n} / P_{0}$ ratio to behave in the limit as a GBM. As shown in the previous section, financial data exhibit strong higher-order moment dependencies, such as asymmetric volatility and conditional heteroskedasticity, which prevent lower frequency log-returns from converging in probability to the normal distribution. These complex dependencies are responsible for the presence of skewness and kurtosis in the data. In this respect, the stochastic process governing price changes is complex and extremely difficult to approximate.

\section{Skewed Generalized Error Distribution}

Introduced by Subbotin (1923), the GED includes as special cases the Laplace, normal, and uniform distributions, e.g., Johnson, Kotz, and Balakrishnan (1995). The GED is used by Box and Tiao (1962) to model prior densities in Bayesian estimation, Nelson (1991) to model the distribution of stock market returns, and Hsieh (1989) to model the distribution of exchange rates.

The GED is nested in the generalized $t$ distribution introduced by 
McDonald and Newey (1988) and its skewed extension introduced by Theodossiou (1998); see also McDonald and Nelson (1989), Butler et al. (1990) and Theodossiou and Savva (2015). Unlike the generalized $t$ distribution, all the moments and the moment generating function of GED exist. Thus, the moments of exponential transformations of GED random variables, needed to price options, can be evaluated.

In this section, the generalized error distribution (GED) is extented to accommodate skewness and leptokurtosis. Its ability to model the empirical distribution of log-returns of financial assets is also evaluated.

\section{A. Skewed GED}

The probability density function for the non-centered SGED is (see appendix A for the derivations)

$$
f_{y}=\frac{k^{1-\frac{1}{k}}}{2 \varphi} \Gamma\left(\frac{1}{k}\right)^{-1} \exp \left(-\frac{1}{k} \frac{|u|^{k}}{(1+\operatorname{sgn}(u) \lambda)^{k} \varphi^{k}}\right)
$$

where $u=y-m, m$ is the mode of the random variable $y, \varphi$ is a scaling constant related to the standard deviation of $y, \lambda$ is a skewness parameter, $k$ is a kurtosis parameter, $s g n$ is the sign function taking the value of -1 for $u<0$ and 1 for $u>0$, and

$$
\Gamma(a)=\int_{0}^{\infty} x^{a-1} e^{-x} d x
$$

is the gamma function. The kurtosis parameter $k$ controls the tails and peakness of the distribution. The skewness parameter $\lambda(-1 \leq \lambda \leq 1)$ controls the rate of descent of the density around $m$. In the case of positive skewness $(\lambda>0)$ the density function is skewed to the right and vice versa.

Its moment function is

where

$$
M_{u, r}=E u^{r}=A_{r} \phi^{r}
$$

$$
A_{r}=\frac{1}{2}\left[(-1)^{r}(1-\lambda)^{r+1}+(1+\lambda)^{r+1}\right] G_{r},
$$




$$
G_{r}=k^{\frac{r}{k}} \Gamma\left(\frac{r+1}{k}\right) \Gamma\left(\frac{1}{k}\right)^{-1} .
$$

The resulting expected value and variance of $y$ are

$$
\mu=E(y)=m+E(u)=m+A_{1} \varphi
$$

and

$$
\sigma^{2}=E u^{2}-(E u)^{2}=\left(A_{2}-A_{1}^{2}\right) \varphi^{2}
$$

where

$$
A_{1}=2 \lambda k^{\frac{1}{k}} \Gamma\left(\frac{2}{k}\right) \Gamma\left(\frac{1}{k}\right)^{-1}
$$

and

$$
A_{2}=\left(1+3 \lambda^{2}\right) k^{\frac{2}{k}} \Gamma\left(\frac{3}{k}\right) \Gamma\left(\frac{1}{k}\right)^{-1} \text {. }
$$

The Pearson's skewness, see Stuart and Ord (1994), p. 108, is

$$
\delta=(\mu-m) / \sigma=A_{1} / \sqrt{A_{2}-A_{1}^{2}} .
$$

The standardized skewness of $y$ is

and

$$
S K=\frac{A_{3}-3 A_{2} A_{1}+2 A_{1}^{3}}{\left(A_{2}-A_{1}^{2}\right)^{3 / 2}}
$$

$$
A_{3}=2 \lambda\left(1+\lambda^{2}\right) k^{\frac{3}{k}} \Gamma\left(\frac{4}{k}\right) \Gamma\left(\frac{1}{k}\right)^{-1} .
$$

The standardized kurtosis is

$$
K U=\frac{A_{4}-4 A_{3} A_{1}+6 A_{2} A_{1}^{2}-3 A_{1}^{4}}{\left(A_{2}-A_{1}^{2}\right)^{2}}
$$

and

$$
A_{4}=\left(1+10 \lambda^{2}+5 \lambda^{4}\right) k^{\frac{4}{k}} \Gamma\left(\frac{5}{k}\right) \Gamma\left(\frac{1}{k}\right)^{-1} .
$$

The SGED gives 1) for $\lambda=0$ the Subbotin's or the power exponential distribution, 2) for $\lambda=0$ and $k=1$ the Laplace or double exponential 


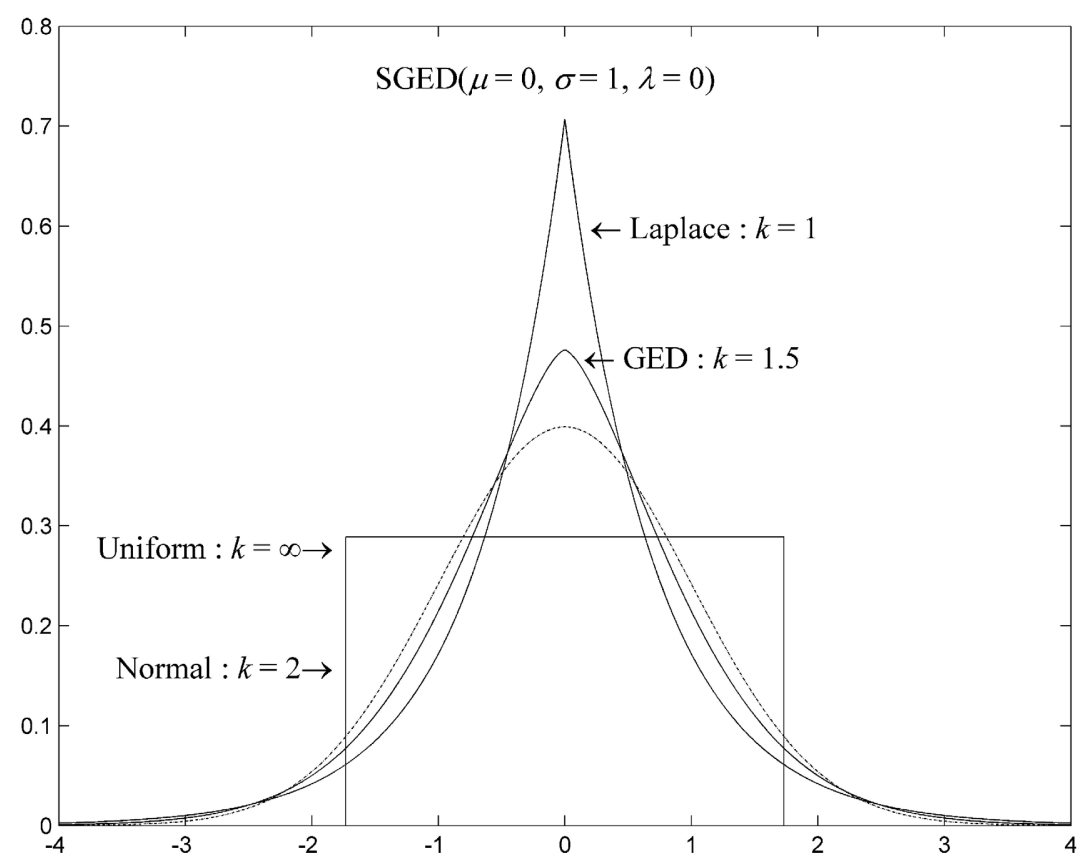

FIGURE 1.- Symmetric GED, Special Cases

distribution, 3) for $k=2$ the skewed normal, 4) for $\lambda=0$ and $k=2$ the normal distribution, 5) for $\lambda=1$ and $k=1$ the exponential distribution and 6) for $\lambda=0$ and $k=\infty$ the uniform distribution. Figure 1 presents a graphical illustration of the GED for a selected combination of the parameters $k$. Note that as $k$ gets smaller, the tails of the distribution become flatter and the center becomes more peaked.

The constants $\varphi$ and $m$ can be expressed in terms of $\sigma$ and $\mu$ as

$$
\varphi=\varphi(\sigma, \lambda, k)=\gamma \sigma
$$

and

$$
m=m(\mu, \sigma, \lambda, k)=\mu-\delta \sigma
$$

where

$$
\gamma=1 / \sqrt{A_{2}-A_{1}^{2}}
$$

$A_{1}, A_{2}$ and $\delta$ (Pearson's skewness) are as defined previously. The direct substitution of the above equations into equation (33) gives the centered 
TABLE 4. Skewness and Kurtosis Relative to $k$ and $\lambda$

\begin{tabular}{clllllll}
\hline \multicolumn{2}{l}{ A. Skewness } & & & & & & \\
$k / \lambda$ & 0.05 & 0.15 & 0.25 & 0.5 & 0.75 & 0.85 & 1 \\
\cline { 2 - 7 } 0.8 & 0.291 & 0.849 & 1.342 & 2.163 & 2.473 & 2.508 & 2.511 \\
0.9 & 0.246 & 0.719 & 1.141 & 1.870 & 2.170 & 2.211 & 2.224 \\
1.0 & 0.212 & 0.620 & 0.989 & 1.644 & 1.935 & 1.979 & 2.000 \\
1.1 & 0.185 & 0.543 & 0.869 & 1.464 & 1.745 & 1.792 & 1.819 \\
1.2 & 0.164 & 0.482 & 0.773 & 1.317 & 1.587 & 1.637 & 1.669 \\
1.3 & 0.146 & 0.431 & 0.694 & 1.194 & 1.455 & 1.506 & 1.542 \\
1.4 & 0.132 & 0.389 & 0.628 & 1.090 & 1.341 & 1.393 & 1.432 \\
1.5 & 0.120 & 0.354 & 0.571 & 1.001 & 1.243 & 1.295 & 1.337 \\
1.6 & 0.109 & 0.323 & 0.523 & 0.923 & 1.157 & 1.209 & 1.253 \\
1.7 & 0.100 & 0.297 & 0.482 & 0.856 & 1.080 & 1.132 & 1.178 \\
1.8 & 0.093 & 0.274 & 0.445 & 0.796 & 1.012 & 1.064 & 1.111 \\
1.9 & 0.086 & 0.254 & 0.413 & 0.742 & 0.951 & 1.002 & 1.050 \\
2.0 & 0.080 & 0.236 & 0.385 & 0.695 & 0.896 & 0.946 & 0.995 \\
5.0 & 0.020 & 0.060 & 0.099 & 0.190 & 0.268 & 0.294 & 0.328 \\
10.0 & 0.006 & 0.019 & 0.031 & 0.061 & 0.090 & 0.100 & 0.115 \\
$\infty$ & 0.000 & 0.000 & 0.000 & 0.000 & 0.000 & 0.000 & 0.000 \\
\hline
\end{tabular}

B. Kurtosis

\begin{tabular}{rrrrrrrr}
$k / \lambda$ & 0.05 & 0.15 & 0.25 & \multicolumn{1}{c}{0.5} & \multicolumn{1}{c}{0.75} & \multicolumn{1}{c}{0.85} & \multicolumn{1}{c}{1} \\
\cline { 2 - 7 } 0.8 & 8.622 & 9.052 & 9.796 & 11.897 & 13.030 & 13.163 & 13.173 \\
0.9 & 7.066 & 7.373 & 7.912 & 9.511 & 10.485 & 10.637 & 10.671 \\
1.0 & 6.030 & 6.258 & 6.664 & 7.920 & 8.765 & 8.922 & 9.000 \\
1.1 & 5.299 & 5.475 & 5.790 & 6.801 & 7.539 & 7.695 & 7.799 \\
1.2 & 4.761 & 4.900 & 5.150 & 5.980 & 6.630 & 6.781 & 6.900 \\
1.3 & 4.351 & 4.462 & 4.666 & 5.357 & 5.934 & 6.079 & 6.205 \\
1.4 & 4.030 & 4.121 & 4.289 & 4.873 & 5.387 & 5.525 & 5.655 \\
1.5 & 3.772 & 3.847 & 3.988 & 4.487 & 4.948 & 5.079 & 5.210 \\
1.6 & 3.561 & 3.625 & 3.744 & 4.175 & 4.590 & 4.714 & 4.844 \\
1.7 & 3.386 & 3.440 & 3.542 & 3.918 & 4.293 & 4.410 & 4.539 \\
1.8 & 3.238 & 3.285 & 3.373 & 3.703 & 4.044 & 4.154 & 4.280 \\
1.9 & 3.113 & 3.153 & 3.230 & 3.522 & 3.833 & 3.937 & 4.060 \\
2 & 3.005 & 3.040 & 3.107 & 3.367 & 3.652 & 3.750 & 3.869 \\
5 & 2.071 & 2.075 & 2.083 & 2.120 & 2.176 & 2.203 & 2.246 \\
10 & 1.884 & 1.886 & 1.888 & 1.899 & 1.917 & 1.927 & 1.942 \\
$\infty$ & 1.800 & 1.800 & 1.800 & 1.800 & 1.800 & 1.800 & 1.800 \\
\hline
\end{tabular}

Note: The above skewness and kurtosis values are computed using equations (35) and (37). Negative values of $\lambda$ generate negative skewness values of equal magnitude as the corresponding positive values. 


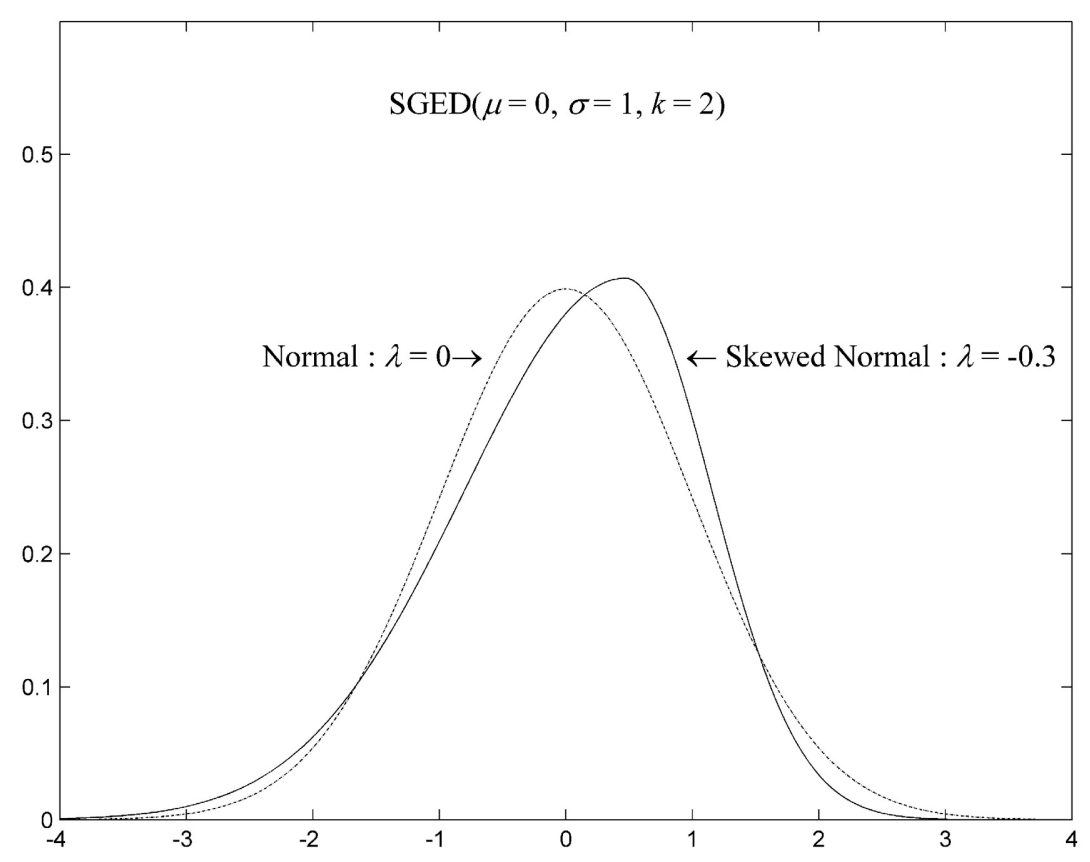

FIGURE 2.- Symmetric and Negatively Skewed Normal Distributions

version of the SGED below

$$
f_{y}=\frac{k^{1-\frac{1}{k}}}{2 \gamma \sigma} \Gamma\left(\frac{1}{k}\right)^{-1} \exp \left(-\frac{1}{k} \frac{|y-\mu+\delta \sigma|^{k}}{(1+\operatorname{sgn}(y-\mu+\delta \sigma) \lambda)^{k} \gamma^{k} \sigma^{k}}\right)
$$

Table 4 presents the skewness and kurtosis values for various combinations of $k$ and $\lambda$. The results provide a better understanding of the relationship between $k$ and $\lambda$ and the shape of the SGED. It appears from the table that smaller values of $k$ generate larger values for the kurtosis; i.e., more leptokurtic density functions. Moreover, larger positive values of $\lambda$ result in larger positive values for both skewness and kurtosis. On the other hand, negative values of $\lambda$ generate identical tables for skewness and kurtosis; however, all skewness values are negative. Figure 2 shows the impact of skewness on the normal density function. 
B. SGED Results

The SGED parameters are obtained by maximizing the log-likelihood function of log-returns $y_{t}$, for $t=1,2, \ldots, T$,

$$
\max _{\vartheta} L\left(\vartheta \mid y_{t}\right)=\sum_{t=1}^{T} \ln f_{y}\left(\vartheta \mid y_{t}\right)
$$

with respect to the parameter vector $\vartheta=[m, \phi, g, k]^{\prime}$, where

$$
\varphi(\phi)=e^{\phi}
$$

and

$$
\lambda(g)=\frac{2}{1+e^{-g}}-1
$$

Both transformations are monotonic functions, therefore there is an one to one relationship between the parameters and their respective transformed values. Moreover, note that the range of $\varphi$ and $g$ is the real line and as such the overshooting problems associated with the constraints imposed on the parameters $\varphi$ and $\lambda$, i.e., $\varphi>0$ and $|\lambda| \leq 1$ is avoided. The non-centered rather than the centered is preferred because of its simplicity

For practical purposes in estimation, the likelihood is specified using the simplified non-centered rather than the centered probability specification. The maximization of the sample log-likelihood function is accomplished using an iterative procedure based on the Berndt, Hall, Hall and Hausman (1974) algorithm. That is, during each iteration, the values for $\theta$ are updated using the equation

$$
\vartheta^{i+1}=\vartheta^{i}+Q\left(\vartheta^{i}\right)^{-1} S\left(\vartheta^{i}\right)
$$

where

$$
S\left(\vartheta^{i}\right)=\frac{\partial L\left(\vartheta^{i}\right)}{\partial \vartheta}=\sum_{t=1}^{T} \frac{\partial L_{t}\left(\vartheta^{i}\right)}{\partial \vartheta}
$$

and

$$
Q\left(\vartheta^{i}\right)=\sum_{t=1}^{T} \frac{\partial L_{t}\left(\vartheta^{i}\right)}{\partial \vartheta} \frac{\partial L_{t}\left(\vartheta^{i}\right)}{\partial \vartheta}
$$


denote the first partial derivatives of the sample log-likelihood and the sum of their cross-products, respectively. These partial derivatives include psi (digamma) functions, computed using numerical techniques. To avoid complications associated with discontinuities in the evaluation of the psi functions, the derivatives are computed numerically using $L$.

The maximum likelihood for the parameters $\varphi$ and $\lambda$ are obtained by substituting the computed MLE values of $\varphi$ and $g$ into equations (51) and (52). Further substitution of the computed values of $m, \varphi, \lambda$ and $k$ into equations (37) and (38) yields the maximum likelihood estimates for the mean $\mu$ and standard deviation $\sigma$ of $y$. Let these MLE estimators

$$
\tilde{\theta}=[\tilde{\mu}, \tilde{\sigma}, \tilde{\lambda}, \tilde{k}]
$$

Their sample variance-covariance matrix can be obtained from

$$
\operatorname{var}(\tilde{\theta})=Q(\tilde{\theta})^{-1}=\sum_{t=1}^{T} \frac{\partial L_{t}(\tilde{\theta})}{\partial \theta} \frac{\partial L_{t}(\tilde{\theta})}{\partial \theta^{\prime}}
$$

or the inverse of the second-cross partial derivatives (Hessian matrix),

$$
\operatorname{var}(\tilde{\theta})=-H(\tilde{\theta})^{-1}=-\sum_{t=1}^{T} \frac{\partial^{2} L(\tilde{\theta})}{\partial \theta \partial \theta^{\prime}} .
$$

Both of the above equations are asymptotically equivalent, because of the known MLE result

$$
E\left(\sum_{t=1}^{T} \frac{\partial L_{t}}{\partial \theta} \frac{\partial L_{t}}{\partial \theta^{\prime}}\right)=-E\left(\sum_{t=1}^{T} \frac{\partial^{2} L_{t}}{\partial \theta \partial \theta^{\prime}}\right)
$$

Robust estimators for the variance-covariance matrix, which is more appropriate in case of mispecification, can be computed using the equation

$$
\operatorname{var}(\theta)=-Q(\tilde{\theta})^{-1} H(\tilde{\theta}) Q(\tilde{\theta})^{-1}
$$

Note that all of the above partial derivatives are computed numerically. Table 5 presents the estimates for the parameters of the SGED for 
TABLE 5. SGED Estimates

\begin{tabular}{|c|c|c|c|c|}
\hline & Daily & Weekly & Monthly & Quarterly \\
\hline \multicolumn{5}{|l|}{ A. Boeing } \\
\hline$\mu_{a}$ & $\begin{array}{l}12.8218 \\
(2.78)^{* *}\end{array}$ & $\begin{array}{l}13.9143 \\
(2.92)^{* *}\end{array}$ & $\begin{array}{l}14.1441 \\
(2.87)^{* *}\end{array}$ & $\begin{array}{l}14.3197 \\
(2.69)^{* * *}\end{array}$ \\
\hline \multirow{2}{*}{$\sigma_{a}$} & 31.0482 & 31.4127 & 31.1321 & 32.7129 \\
\hline & $(116.74)^{* *}$ & $(55.78)^{* *}$ & $(27.67)^{* *}$ & $(13.21)^{* *}$ \\
\hline \multirow[t]{2}{*}{$k$} & 1.1246 & 1.2996 & 1.3575 & 1.3393 \\
\hline & $(114.34)^{* *}$ & $(41.7)^{* *}$ & $(12.43)^{* *}$ & $(5.81)^{* *}$ \\
\hline \multirow[t]{2}{*}{$\lambda$} & 0.0347 & -0.0053 & -0.0858 & -0.1618 \\
\hline & $(7.31)^{* *}$ & $(-0.26)$ & $(-1.45)$ & $(-1.18)$ \\
\hline$\delta$ & 0.0504 & -0.008 & -0.1291 & -0.2413 \\
\hline$S K$ & 0.1245 & -0.0156 & -0.2352 & -0.4448 \\
\hline$K U$ & 5.14 & 4.34 & 4.18 & 4.34 \\
\hline $\log L$ & $-20,605.2$ & $-5,982.25$ & $-1,721.1$ & -665.19 \\
\hline$L R$ & $1380.01 * *$ & $143.8^{* *}$ & $25.78^{* *}$ & $7.69 *$ \\
\hline Observations & 10,091 & 2,087 & 479 & 159 \\
\hline \multicolumn{5}{|l|}{ B. IBM } \\
\hline \multirow[t]{2}{*}{$\mu_{a}$} & 5.0837 & 4.5339 & 4.5133 & 4.914 \\
\hline & $(1.34)$ & $(1.13)$ & $(1.19)$ & $(0.93)$ \\
\hline \multirow[t]{2}{*}{$\sigma_{a}$} & 25.6804 & 26.5179 & 24.8387 & 25.8231 \\
\hline & $(115.2)^{* *}$ & $(53.13)^{* *}$ & $(28.27)^{* *}$ & $(14.82)^{* *}$ \\
\hline \multirow[t]{2}{*}{$k$} & 1.0525 & 1.0795 & 1.4139 & 1.4782 \\
\hline & $(96.91)^{* *}$ & $(37.04)^{* *}$ & $(11.53)^{* *}$ & $(4.45)^{* *}$ \\
\hline \multirow[t]{2}{*}{$\lambda$} & 0.0077 & 0.0117 & 0.0432 & -0.0174 \\
\hline & $(1.2)$ & $(1.28)$ & $(1.18)$ & $(-0.07)$ \\
\hline$\delta$ & 0.011 & 0.0169 & 0.0657 & -0.0266 \\
\hline$S K$ & 0.0302 & 0.0447 & 0.1126 & -0.0425 \\
\hline$K U$ & 5.59 & 5.41 & 3.99 & 3.81 \\
\hline $\log L$ & $-18,563.37$ & $-5,564.74$ & $-1,615.86$ & -630.59 \\
\hline$L R$ & $1,904.44 * *$ & $334.39 * *$ & $18.61^{* *}$ & 4.89 \\
\hline Observations & 10,091 & 2,087 & 479 & 159 \\
\hline \multicolumn{5}{|l|}{ C. DJIA } \\
\hline \multirow[t]{2}{*}{$\mu_{a}$} & 9.0953 & 8.2669 & 8.5374 & 8.1550 \\
\hline & $(2.9)^{* *}$ & $(3.16)^{* *}$ & $(3.56)^{* *}$ & $(3.11)^{* *}$ \\
\hline \multirow[t]{2}{*}{$\sigma_{a}$} & 16.4907 & 16.9915 & 15.3405 & 15.9583 \\
\hline & $(109.88)^{* *}$ & $(51.34)^{* *}$ & $(26.14)^{* *}$ & $(13.93)^{* *}$ \\
\hline \multirow[t]{2}{*}{$k$} & 0.9379 & 1.1378 & 1.2303 & 1.3822 \\
\hline & $(147.94)^{* *}$ & $(52.26)^{* *}$ & $(13.9)^{* *}$ & $(5.68)^{* *}$ \\
\hline \multirow[t]{2}{*}{$\lambda$} & 0.0364 & -0.0524 & -0.1795 & -0.2396 \\
\hline & $(2.6)^{* *}$ & $(-2.44)^{*}$ & $(-3.79)^{* *}$ & $(-1.65)$ \\
\hline$\delta$ & 0.0506 & -0.0762 & -0.2625 & -0.3556 \\
\hline
\end{tabular}


TABLE 5. (Continued)

\begin{tabular}{|c|c|c|c|c|}
\hline & Daily & Weekly & Monthly & Quarterly \\
\hline \multicolumn{5}{|l|}{ C. DJIA } \\
\hline$S K$ & 0.1688 & -0.1846 & -0.5517 & -0.6145 \\
\hline$K U$ & 6.61 & 5.08 & 4.81 & 4.33 \\
\hline $\log L$ & $-14,075.35$ & $-4,654.71$ & $-1,371.18$ & -549.95 \\
\hline$L R$ & $2,655.04 * *$ & $348.16^{* *}$ & $47.55^{* *}$ & $14.07 * *$ \\
\hline Observations & 10,435 & 2,087 & 479 & 159 \\
\hline \multicolumn{5}{|l|}{ D. S\&P100 } \\
\hline$\mu_{a}$ & $\begin{array}{l}10.1215 \\
(1.2)\end{array}$ & $\begin{array}{c}7.9407 \\
(2.51)^{*}\end{array}$ & $\begin{array}{c}7.9296 \\
(2.77)^{* *}\end{array}$ & $\begin{array}{c}7.8124 \\
(2.63)^{* *}\end{array}$ \\
\hline \multirow[t]{2}{*}{$\sigma_{a}$} & 18.0505 & 17.6638 & 15.8855 & 16.6634 \\
\hline & $(107.51)^{* *}$ & $(45.06)^{* *}$ & $(20.22)^{* *}$ & $(9.64)^{* *}$ \\
\hline \multirow[t]{2}{*}{$k$} & 0.8563 & 1.033 & 1.1542 & 1.1354 \\
\hline & $(124.32)^{* *}$ & $(43.18)^{* *}$ & $(11.39)^{* *}$ & $(4.1)^{* *}$ \\
\hline \multirow[t]{2}{*}{$\lambda$} & 0.0319 & -0.052 & -0.2186 & -0.2688 \\
\hline & $(0.81)$ & $(-4.55)^{* *}$ & $(-4.95) * *$ & $(-4.33)^{* *}$ \\
\hline$\delta$ & 0.0432 & -0.074 & -0.3132 & -0.3797 \\
\hline$S K$ & 0.1685 & -0.2099 & -0.7223 & -0.887 \\
\hline$K U$ & 7.64 & 5.76 & 5.32 & 5.61 \\
\hline $\log L$ & $-11,330.51$ & $-3,616.54$ & $-1,062.64$ & -425.98 \\
\hline$L R$ & $2,504.08 * *$ & $345.73^{* *}$ & $50.5 * *$ & $20.43^{* *}$ \\
\hline Observations & 8,042 & 1,608 & 369 & 123 \\
\hline \multicolumn{5}{|l|}{ E. S\&P500 } \\
\hline$\mu_{a}$ & $\begin{array}{l}9.6742 \\
(1.09)\end{array}$ & $\begin{array}{c}8.4355 \\
(3.22)^{* *}\end{array}$ & $\begin{array}{c}8.5264 \\
(3.52)^{* *}\end{array}$ & $\begin{array}{c}8.3945 \\
(3.28)^{* *}\end{array}$ \\
\hline \multirow[t]{2}{*}{$\sigma_{a}$} & 16.7313 & 17.154 & 15.3151 & 16.2089 \\
\hline & $(94.37)^{* *}$ & $(52.4)^{* *}$ & $(26.55)^{* *}$ & $(13.3)^{* *}$ \\
\hline \multirow[t]{2}{*}{$k$} & 0.9012 & 1.1012 & 1.2474 & 1.3726 \\
\hline & $(73.25)^{* *}$ & $(48.1)^{* *}$ & $(12.69)^{* *}$ & $(5.01)^{* *}$ \\
\hline \multirow[t]{2}{*}{$\lambda$} & 0.029 & -0.0668 & -0.2028 & -0.2998 \\
\hline & $(0.93)$ & $(-4.67)^{* *}$ & $(-4.04)^{* *}$ & $(-3.73)^{* *}$ \\
\hline$\delta$ & 0.0399 & -0.0964 & -0.2964 & -0.4394 \\
\hline$S K$ & 0.1426 & -0.2461 & -0.6064 & -0.7559 \\
\hline$K U$ & 7.02 & 5.31 & 4.79 & 4.49 \\
\hline $\log L$ & $-14,097.67$ & $-4,659.94$ & $-1,369.8$ & -550.54 \\
\hline$L R$ & $2,876.27 * *$ & $357.91 * *$ & $51.56^{* *}$ & $16.71 * *$ \\
\hline Observations & 10,435 & 2,087 & 479 & 159 \\
\hline \multicolumn{5}{|l|}{ F. GBP/USD } \\
\hline \multirow[t]{2}{*}{$\mu_{a}$} & -1.0656 & -1.0781 & -1.2184 & -0.9716 \\
\hline & $(-0.86)$ & $(-0.68)$ & $(-0.75)$ & $(-0.56)$ \\
\hline \multirow[t]{2}{*}{$\sigma_{a}$} & 9.6222 & 10.1237 & 10.3942 & 10.5671 \\
\hline & $(99.17)^{* *}$ & $(53.8)^{* *}$ & $(27.57)^{* *}$ & $(12.43)^{* *}$ \\
\hline
\end{tabular}


TABLE 5. (Continued)

\begin{tabular}{|c|c|c|c|c|}
\hline & Daily & Weekly & Monthly & Quarterly \\
\hline \multicolumn{5}{|l|}{ F. GBP/USD } \\
\hline \multirow[t]{2}{*}{$k$} & 1.0037 & 1.1881 & 1.4258 & 1.2525 \\
\hline & $(66.03)^{* *}$ & $(31.92)^{* *}$ & $(14.19)^{* *}$ & $(5.89)^{* *}$ \\
\hline \multirow[t]{2}{*}{$\lambda$} & 0.0025 & -0.0603 & -0.0436 & -0.1139 \\
\hline & $(0.15)$ & $(-2.48)^{*}$ & $(-1.05)$ & $(-0.64)$ \\
\hline$\delta$ & 0.0036 & -0.0886 & -0.0663 & -0.1685 \\
\hline$S K$ & 0.0107 & -0.1999 & -0.1121 & -0.3478 \\
\hline$K U$ & 5.97 & 4.83 & 3.96 & 4.6 \\
\hline $\log L$ & $-8,649.89$ & $-3,588.95$ & $-1,198.95$ & -484.86 \\
\hline$L R$ & $1,524.82 * *$ & $195.51^{* *}$ & $19.85^{* *}$ & $10.82 * *$ \\
\hline Observations & 10,435 & 2,087 & 479 & 159 \\
\hline \multicolumn{5}{|l|}{ G. JY/USD } \\
\hline \multirow[t]{2}{*}{$\mu_{a}$} & -1.9077 & -1.803 & -1.8054 & -1.9732 \\
\hline & $(-0.92)$ & $(-0.87)$ & $(-0.82)$ & $(-0.82)$ \\
\hline \multirow[t]{2}{*}{$\sigma_{a}$} & 11.1756 & 11.3946 & 11.4521 & 12.4452 \\
\hline & $(93.48)^{* *}$ & $(47.85)^{* *}$ & $(22.74)^{* *}$ & $(12.44)^{* *}$ \\
\hline \multirow[t]{2}{*}{$k$} & 1.0207 & 1.2743 & 1.4376 & 1.6835 \\
\hline & $(68.31)^{* *}$ & $(32.13)^{* *}$ & $(11.18)^{* *}$ & $(4.57)^{* *}$ \\
\hline \multirow[t]{2}{*}{$\lambda$} & -0.005 & -0.1182 & -0.0664 & -0.2529 \\
\hline & $(-0.86)$ & $(-4.58)^{* *}$ & $(-0.81)$ & $(-2.04)^{*}$ \\
\hline$\delta$ & -0.0071 & -0.1754 & -0.1011 & -0.3879 \\
\hline$S K$ & -0.0207 & -0.352 & -0.1686 & -0.4933 \\
\hline$K U$ & 5.83 & 4.52 & 3.93 & 3.58 \\
\hline $\log L$ & $-7,422.2$ & $-2,790.65$ & -902.15 & -371.01 \\
\hline$L R$ & $1,244.32 * *$ & $124.14^{* *}$ & $11.09^{* *}$ & 4.07 \\
\hline Observations & 7,564 & 1,513 & 347 & 115 \\
\hline
\end{tabular}

Note: The SGED parameters are obtained by maximizing the sample log-likelihood function with respect to the parameters $\mu, \sigma, k$, and $\lambda$, where $C, \theta$, and $\delta$ are given by equations (11)-(13), sign is the sign of the residual $\left(y_{t}-\mu+\delta \sigma\right), T$ is the sample size, $\ln$ is the natural logarithm. The statistics for the means and standard deviations are annualized using $\Delta t=$ $1 / 252,1 / 52,1 / 12,1 / 6$, and $1 / 4$ for the daily, weekly, monthly, and quarterly log-returns. The estimate for $\delta$ (Pearson's skewness) is computed by substituting the estimates for $\lambda$ and $k$ into equation (13). $L R$ is the log-likelihood ratio statistics for testing the null-hypothesis that the series follow the normal distribution against the alternative hypothesis of the SGED distribution. The $L R$ statistic follows the $\chi^{2}(2)$ with two degrees of freedom. *** imply statistically significant the five- and one-percent level of significance, respectively.

all log-return series and frequencies. The first eight rows report the estimates and $t$-values for the mean, standard deviation, kurtosis, and skewness parameters of the SGED. The $t$-values for these estimates are enclosed in parentheses below the estimates. As in table 2, the estimated means and standard deviations of log-returns are annualized using the 


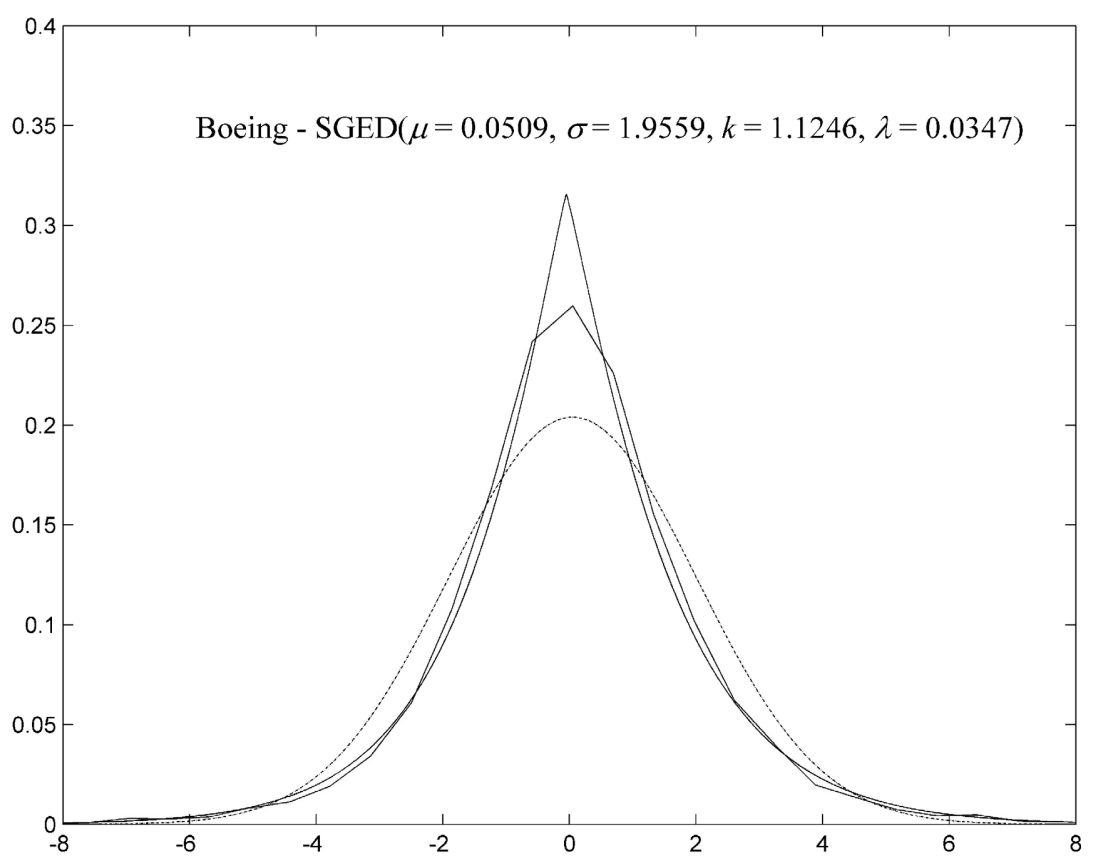

FIGURE 3.- Boeing, Empirical Distributions of Log-Returns

formulas $\tilde{\mu}_{a}=\tilde{\mu} / \Delta t$ and $\tilde{\sigma}_{a}=\tilde{\sigma} / \sqrt{\Delta t}$, where $\Delta t=1 / 252,1 / 52,1 / 12$ and $1 / 4$ for daily, weekly, monthly and quarterly log-returns, respectively. The remaining rows contain estimates of Pearson's skewness $\delta$, skewness $S K$ and kurtosis $K U$ calculated using equations (34), (35) and (37), the sample $\log$-likelihood function $(\log L)$, and the $\log$-likelihood ratio $(L R)$ for testing the null hypothesis of normality against the alternative hypothesis of the SGED.

The estimated means and standard deviations of each series are similar across the four data frequencies and close to the respective estimates in table 2 . The estimates of the kurtosis parameter $k$ ranges between 0.8563 and 1.1246 for daily, 1.033 and 1.2996 for weekly, 1.1542 and 1.4376 for monthly and 1.1354 and 1.6835 for quarterly logreturns. Its respective means across the four frequencies are 0.9853 , $1.1591,1.3238$ and 1.3777. These numbers indicate that, on average, daily log-returns follow the Laplace distribution. The other log-return data frequencies diverge from the Laplace distribution but do not become normal $(k=2)$. 


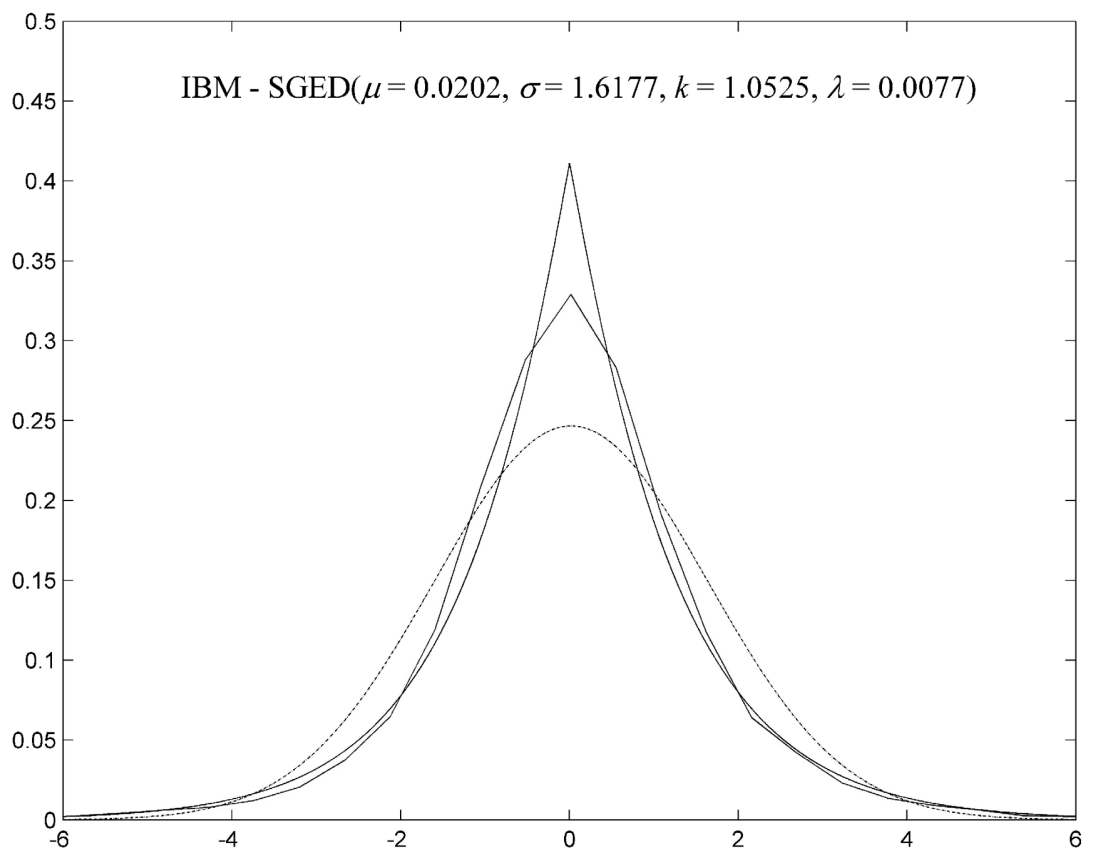

FIGURE 4.- IBM, Empirical Distributions of Log-Returns

The kurtosis $K U$ values range between 5.14 and 7.64 for daily, 4.34 and 5.76 for weekly, 3.93 and 5.32 for monthly and 3.58 and 5.61 for quarterly log-returns. Their respective means across the four frequencies are $6.26,5.03,4.43$ and 4.39 . These numbers also indicate that, on average, daily log-returns have a kurtosis value of 6.26 close to that Laplace distribution $(K U=6)$. The kurtosis values of the remaining logreturn frequencies are smaller, but do not approach the kurtosis of the normal distribution $(K U=3)$.

The results for skewness $(\lambda)$ indicate, in general, the presence of negative skewness in the data. Twenty out of the twenty-eight estimated values of $\lambda$ are negative. In eleven of these cases the estimates are statistically significant. In eight cases the estimates of $\lambda$ are positive but only in two of them are statistically significant. The results for skewness are consistent with those of table 2 .

Because the normal distribution is nested under the SGED, a loglikelihood ratio statistic could be used to test the null hypothesis that log-returns are normally distributed against the alternative hypothesis 


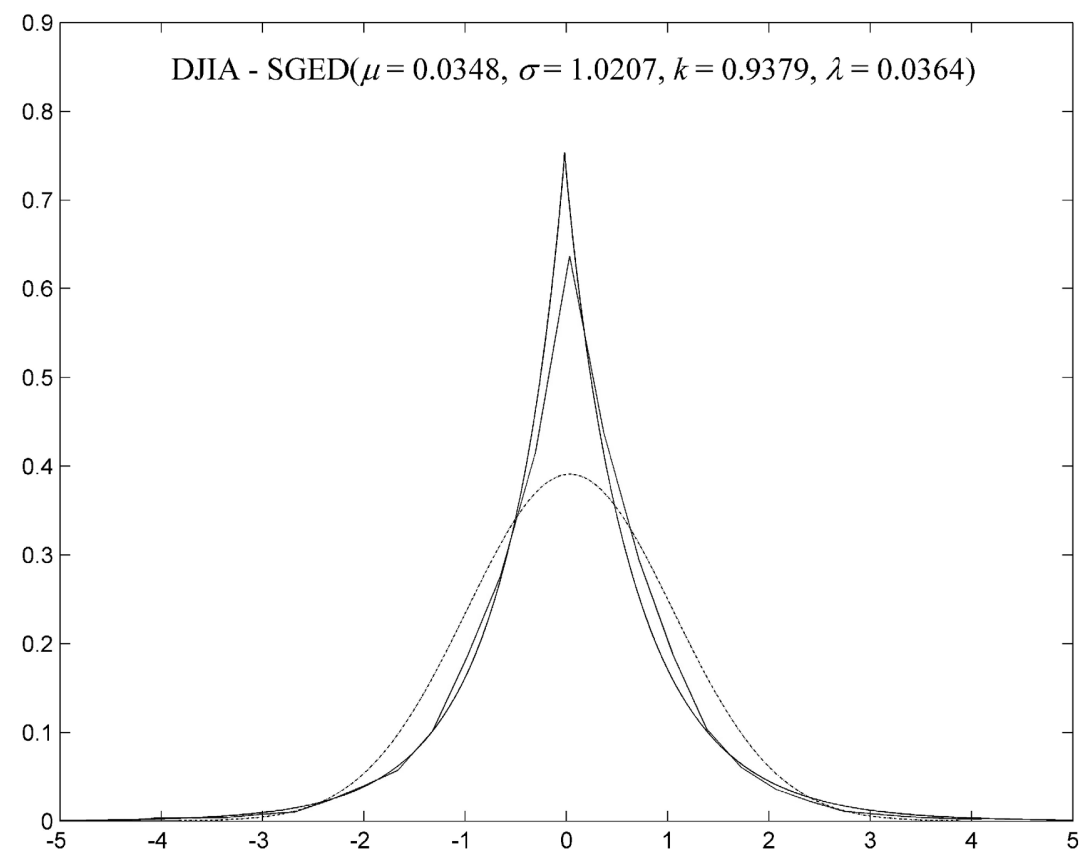

FIGURE 5.- DJIA, Empirical Distributions of Log-Returns

that they are SGED distributed. The null hypothesis for testing normality is $H_{0}: k=2$ and $\lambda=0$ and the alternative hypothesis is $H_{1}: k$ $\in R^{+}$and $|\lambda| \leq 1$. The statistic is computed using the formula $L R=$ $-2 \times\left(\log L_{0}-\log L\right)$, where $\log L_{0}$ is the maximum value of the $\log -$ likelihood function under the null hypothesis of normality and $\log L$ is the maximum value of the log-likelihood function under the SGED specification. The $L R$ statistic is asymptotically distributed as a chisquare with two degrees of freedom, $\chi^{2}(2)$. With one exception (quarterly log-returns for IBM), the $L R$ statistics reject the null hypothesis of normality. Note that for a small sample the statistical power of the $L R$ statistic is weak and may accept the null hypothesis of normality when it should be rejected.

Figures 3 to 9 present the empirical distributions for the daily logreturns of the seven series. The figures for weekly, monthly and quarterly log-returns are qualitatively similar, therefore, are omitted. The estimated SGED distributions are represented by the solid curves and the estimated non-parametric distributions by the piecewise linear 


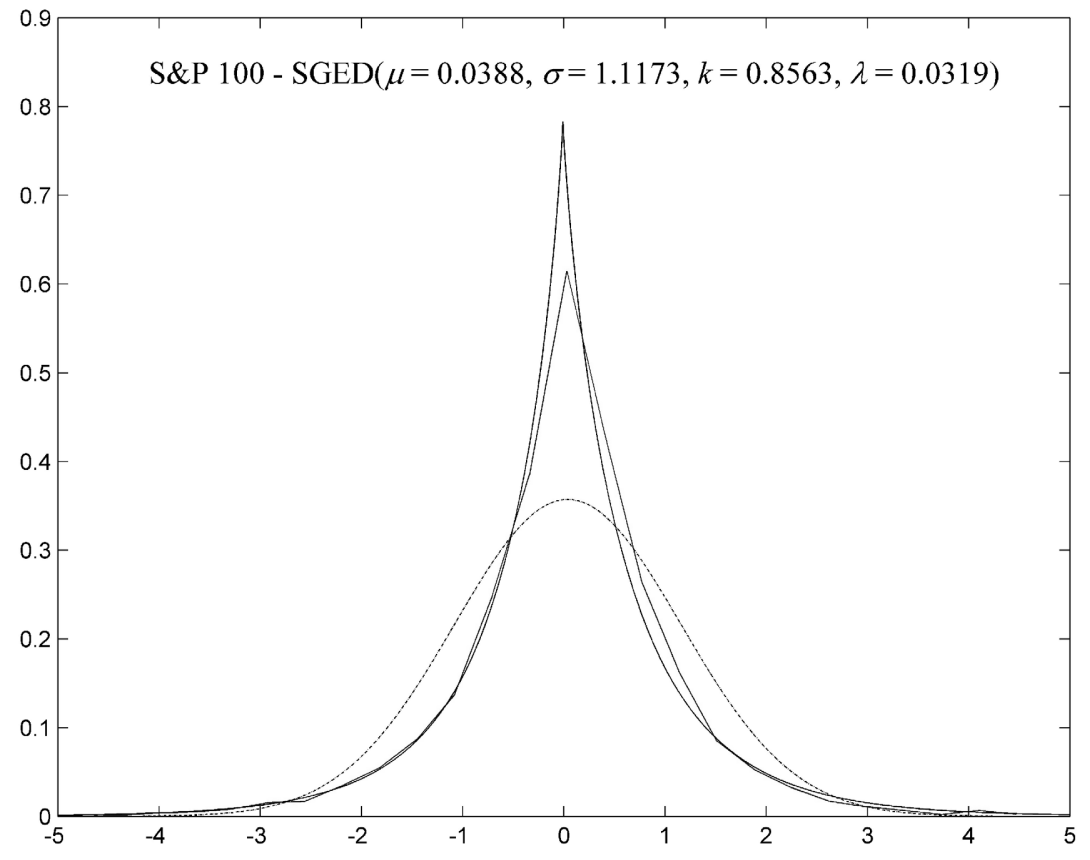

FIGURE 6.- S\&P 100, Empirical Distributions of Log-Returns

curves; see Tapia and Thompson (1978), section 2.5 for the latter. For comparison purposes, the estimated normal distributions are also presented by the dotted curves. These graphs demonstrate that the SGED provides a very good fit to the empirical distribution of logreturns of all series. In some cases, the estimated SGED and the nonparametric probability curves are indistinguishable. Moreover, they deviate significantly from those of the normal distribution.

\section{Call Option Pricing}

This section derives the SGED option pricing model and demonstrates its relationship to several well-known pricing models for European call options. These are the Black and Scholes' (1973) model, Merton's (1973) continuous dividend yield model, Biger and Hull's (1983) currency model, and Black's (1976) currency futures model. 


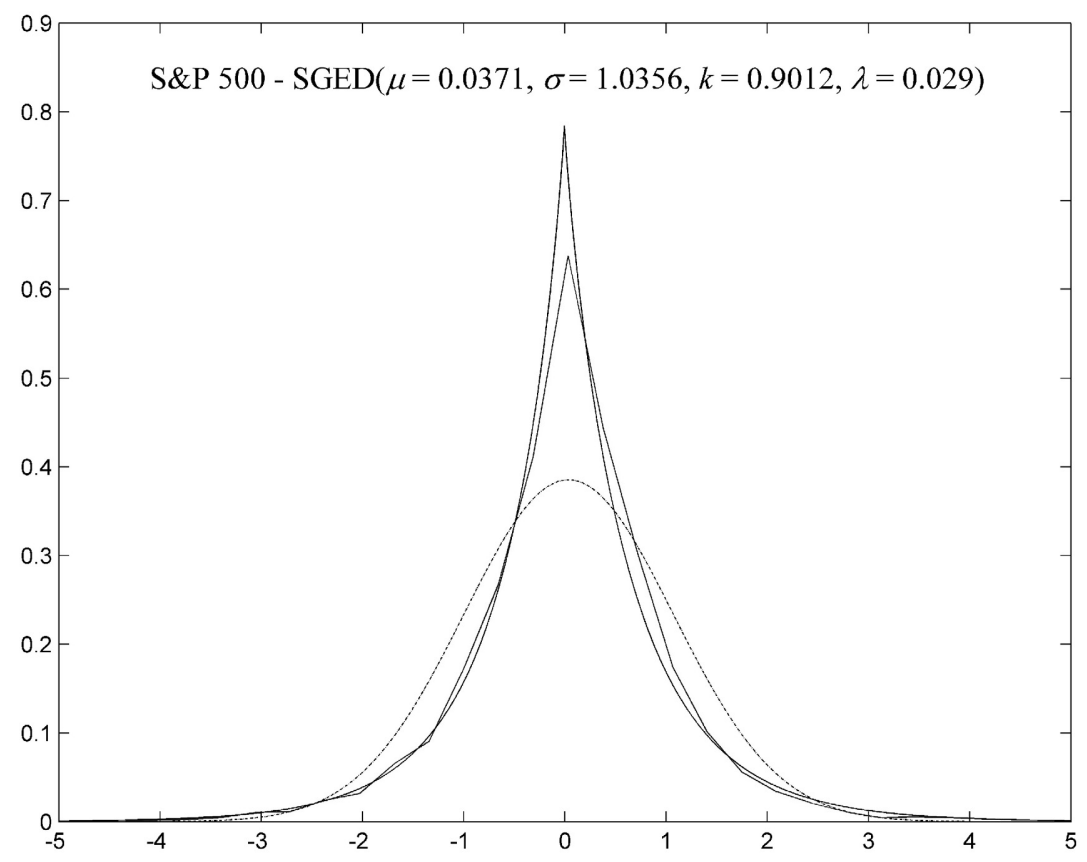

FIGURE 7.- S\&P 500, Empirical Distributions of Log-Returns

\section{A. SGED Option Pricing Model}

Consider a European call option with a strike price $X$ written on an asset with a current (spot) price $P_{0}$ that pays no dividends and expires $n$ periods from time $t=0$. Let $\tau=n \Delta t$ be the option's time to expiration. The value of this option at expiration is equal to $C_{n}=\max \left(P_{n}-X, 0\right)$, where $P_{n}$ is the price of the underlying asset at expiration. Observe that

$$
P_{n}=P_{0} \exp \left(\ln \left(P_{n} / P_{0}\right)\right)=P_{0} e^{Y_{n}}
$$

where

$$
Y_{n}=\ln \left(P_{n} / P_{0}\right)=\mu_{a} \tau+z \sigma_{a} \sqrt{\tau}
$$

is the log-return to prevail over the life of the option as a function of the mean and the standard deviation of $Y_{n}$ and the standardized random variable $z$ with probability mass function given by $d F(z)=f(z) d z$. 


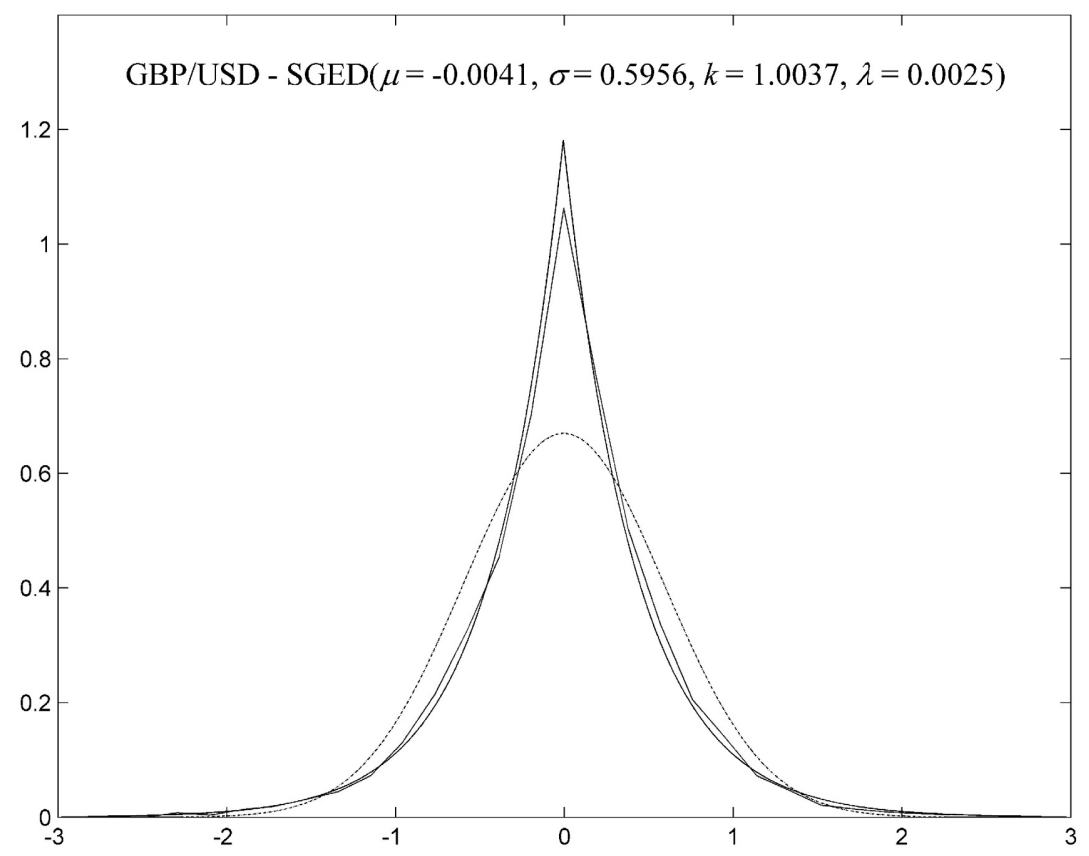

FIGURE 8.- GBP/USD, Empirical Distributions of Log-Returns

The expected value of the option at time $n$ is

$$
\begin{aligned}
& E\left(C_{n}\right)=E\left(\max \left(P_{n}-X, 0\right)\right) \\
& =P_{0} E\left(\max \left(e^{Y_{n}}-X / P_{0}, 0\right)\right) .
\end{aligned}
$$

The presence of a positive pay-off at expiration (i.e., $P_{n}>X$ ) implies that

$$
Y_{n}=\mu_{a} \tau+z \sigma_{a} \sqrt{\tau}>-\ln \left(P_{0} / X\right)
$$

or

$$
z>-\frac{\ln \left(P_{0} / X\right)+\mu_{a} \tau}{\sigma_{a} \sqrt{\tau}} \equiv h .
$$

The function $E\left(C_{n}\right)$ can be expressed in terms of $z$ 


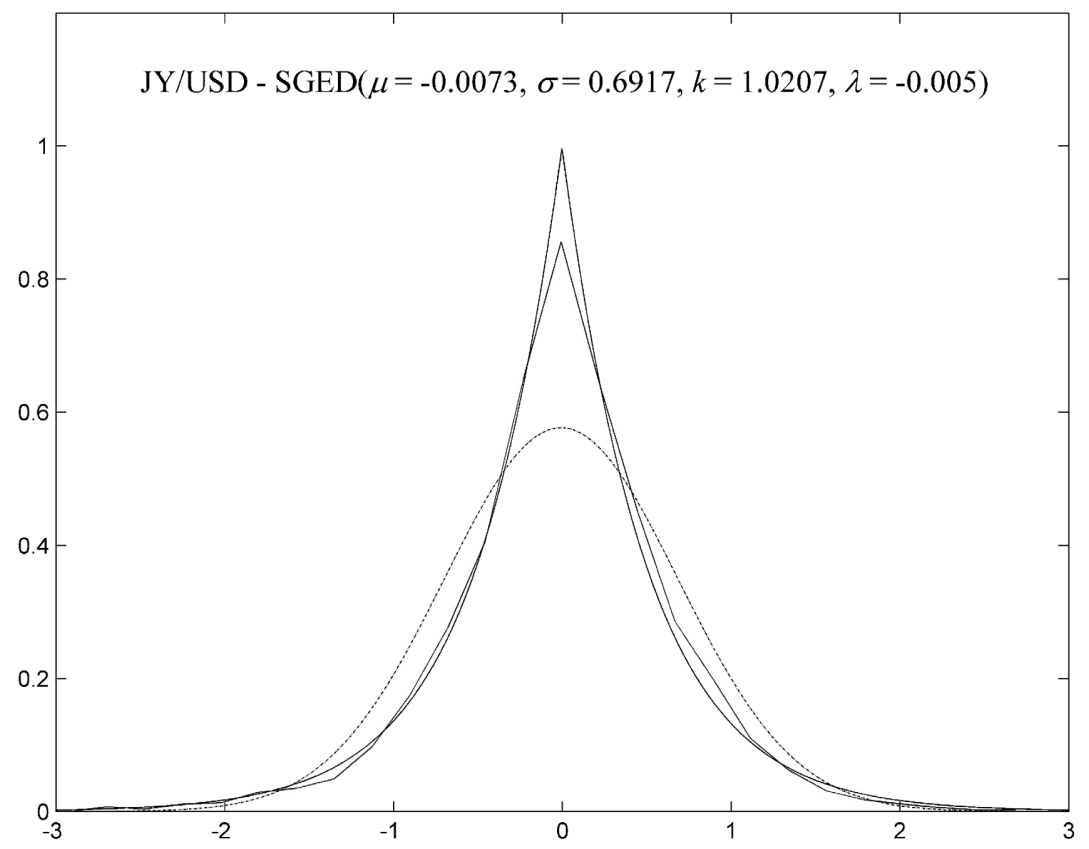

FIGURE 9.- JY/USD, Empirical Distributions of Log-Returns

$$
\begin{aligned}
& E\left(C_{n}\right)=P_{0} \int_{h}^{\infty}\left(e^{\mu_{a} \tau+z \sigma_{a} \sqrt{\tau}}-X / P_{0}\right) d F(z) \\
& =P_{0} e^{\mu_{a} \tau} \int_{h}^{\infty} e^{z \sigma_{a} \sqrt{\tau}} d F_{z}(z)-X \int_{h}^{\infty} d F_{z}(z) \\
& =P_{0} e^{\mu_{a} \tau} \int_{h}^{\infty} e^{z \sigma_{a} \sqrt{\tau}} d F_{z}(z)-X\left(1-F_{z}(h)\right)
\end{aligned}
$$

where $h$ is as defined above.

The option price at $t=0$ is equal to present value of $E\left(C_{n}\right)$. That is,

$$
C_{0}=e^{-r \tau} E\left(C_{n}\right)
$$




$$
=P_{0} e^{\left(\mu_{a}-r\right) \tau} \int_{h}^{\infty} e^{z \sigma_{a} \sqrt{\tau}} d F_{z}(z)-e^{-r \tau} X\left(1-F_{z}(h)\right)
$$

where $r$ is an equilibrium discount rate of return for buyers and issuers of call options. Under risk neutrality, this discount rate is taken to be equal to the risk free rate. In the above equation, the parameters $k$, and $\lambda$ could be obtained by maximizing the SGED sample log-likelihood function, equation 50, using data that best reflect the distribution of logreturns of the underlying asset during the life of the option. As for $\sigma_{\alpha}$ measures of implied volatility may be used. The values of $P_{0}$ and $X$ are always known and do not change over the life of the call option.

\section{B. Special Cases of SGED Option Pricing Model}

Under normality, the integral in equation (36), see appendix B, becomes

$$
\int_{h}^{\infty} e^{z \sigma_{a} \sqrt{\tau}} f(z) d z=e^{\frac{1}{2} \sigma_{a}^{2} \tau} \Phi\left(-h+\sigma_{a} \sqrt{\tau}\right)=e^{\frac{1}{2} \sigma_{a}^{2} \tau} N\left(d_{1}\right)
$$

where

$$
d_{1} \equiv-h+\sigma_{a} \sqrt{\tau}=\frac{\ln \left(P_{0} / X\right)+\left(\mu_{a}+\sigma_{a}^{2}\right) \tau}{\sigma_{a} \sqrt{\tau}} .
$$

Moreover, the probability,

$$
1-F_{z}(h)=1-\Phi(h)=\Phi(-h)=N\left(d_{2}\right),
$$

where

$$
d_{2}=d_{1}-\sigma_{a} \sqrt{\tau}
$$

Note that $d_{1}$ and $d_{2}$ is standard notation used in option pricing models and $N$ is used insetad of $\Phi$. Substitution of these quantities into the option pricing equation of equation (65) yields

$$
C_{0}=P_{0} e^{\left(\mu_{a}+\frac{1}{2} \sigma_{a}^{2}-r\right) \tau} N\left(d_{1}\right)-e^{-r \tau} X N\left(d_{2}\right)
$$

which can be used as pricing kernel in cases where log-returns are 
normal or returns are log-normal. In the above equation, the quantity $\mu_{a}+\frac{1}{2} \sigma_{2}^{2}$ measures the stock's expected return; see equation (27).

\section{Black and Scholes Model}

In the absence of arbitrage opportunities, the expected annual return of a stock paying no dividend is equal to the risk-free rate. That is,

$$
\mu_{a}+\frac{1}{2} \sigma_{a}^{2}=r
$$

Substitution of this equality into equation (70) gives the Black and Scholes (1973) option pricing model,

$$
C_{0}=P_{0} N\left(d_{1}\right)-e^{-r \tau} X N\left(d_{2}\right)
$$

where

$$
d_{1}=\frac{\ln \left(P_{0} / X\right)+\left(r+\frac{1}{2} \sigma_{a}^{2}\right) \tau}{\sigma_{a} \sqrt{\tau}}
$$

and $d_{2}=d_{1}-\sigma_{a} \sqrt{\tau}$.

\section{Merton's Model}

In the case of a stock paying continuous dividend at an annual rate $q$, the expected stock return, including dividends, is equal to the risk free borrowing rate $r$. That is,

$$
\mu_{a}+\frac{1}{2} \sigma_{a}^{2}+q=r
$$

Substitution of this equality into equation (70) gives Merton's (1973) continuous dividend yield model,

$$
C_{0}=P_{0} e^{-q \tau} N\left(d_{1}\right)-e^{-r \tau} X N\left(d_{2}\right),
$$

where

$$
d_{1}=\frac{\ln \left(P_{0} / X\right)+\left(r-q+\frac{1}{2} \sigma_{a}^{2}\right) \tau}{\sigma_{a} \sqrt{\tau}}
$$

and $d_{2}=d_{1}-\sigma_{a} \sqrt{\tau}$. 


\section{Currency Option Pricing Model}

In the absence of arbitrage opportunities, the expected growth rate of a currency is equal to the difference between the domestic $r$ and foreign interest rates $r_{f}$. That is,

$$
\mu_{a}+\frac{1}{2} \sigma_{a}^{2}=r-r_{f}
$$

Substitution of this equality into equation (70) gives Biger and Hull's (1983) currency option pricing model,

where

$$
C_{0}=P_{0} e^{-r_{f} \tau} N\left(d_{1}\right)-e^{-r \tau} X N\left(d_{2}\right),
$$

$$
d_{1}=\frac{\ln \left(P_{0} / X\right)+\left(r-r_{f}+\frac{1}{2} \sigma_{a}^{2}\right) \tau}{\sigma_{a} \sqrt{\tau}}
$$

and $d_{2}=d_{1}-\sigma_{a} \sqrt{\tau}$.

\section{Currency Future Option Model}

Another variation of the model above is obtained by substituting the exchange rate parity

$$
F_{n}=P_{0} e^{\left(r-r_{f}\right) \tau},
$$

where $F_{n}$ is the forward exchange rate into equation (70). That is,

$$
C_{0}=e^{-r \tau}\left[F_{n} N\left(d_{1}\right)-X N\left(d_{2}\right)\right]
$$

where

and $d_{2}=d_{1}-\sigma_{a} \sqrt{\tau}$.

$$
d_{1}=\frac{\ln \left(F_{n} / X\right)+\frac{1}{2} \sigma_{a}^{2} \tau}{\sigma_{a} \sqrt{\tau}}
$$

This formulation is similar to Black's (1976) model for pricing options on currency futures, where $F_{n}$ represents the futures price.

\section{General Comments}

Note that the four variation of the option pricing model presented above 
are based on the assumptions of normality of log-returns and absence of arbitrage opportunities or that the mean return for stocks and stock indices is equal to the risk free rate and for currencies equal to the spread between the domestic and foreign interest rates. Table 5 presents estimates for the mean returns of the seven series. The means of all stocks and stock indices are considerably higher from the U.S. t-bill rate, which has ranged between 3 and 6 percent during the past ten years. The mean returns, however, for the two currencies are close to the spread between the t-bill rate in the U.S. and those in the U.K. and Japan. These differences are indirectly compensated with the use of implied volatility measures which are typically larger than those obtained through estimation using historical data.

\section{Summary and Concluding Remarks}

Analytical formulas and test statistics developed in this article established that the presence of skewness and kurtosis in financial data was the result of higher-order moment dependencies, such as conditional heteroskedasticity, asymmetric volatility, and other nonlinear dependencies, that existed mainly in daily and weekly log-returns. These dependencies prevented monthly, bimonthly, and quarterly logreturns to obey the normality law implied by the central limit theorem. As a consequence, prices of these assets violated the geometric Brownian motion often assumed in pricing options and other derivative assets.

The above findings necessitated the development of distributions that provided better approximations to the empirical distributions of logreturns. This article developed a skewed extension of the generalized error distribution (SGED) and assessed its ability to fit the empirical distribution of log-returns of several popular financial assets at various data frequencies. The results showed that the SGED provided a good fit to the empirical distribution of the data.

A model for pricing European call options based on the SGED was developed that accommodated skewness and kurtosis in the data and relaxed the assumptions of risk neutrality and no arbitrage opportunities. Nevertheless, under the standard assumptions in option pricing of normality of log-returns, risk neutrality, and absence of arbitrage opportunities, the SGED model produced as special cases several wellknown option pricing models, such as the Black and Scholes (1973) model, Merton's (1973) continuous dividend yield model, Biger and 
Hull's (1983) currency model, and Black's (1976) currency futures model.

\section{Appendix A. SGED's Moments}

Consider the non-centered SGED probability density function

$$
f(u)=C \exp \left(-\frac{1}{k} \frac{|u|^{k}}{(1+\operatorname{sgn}(u) \lambda)^{k} \varphi^{k}}\right),
$$

where $C$ is a scaling constant. The $r$ th non-centered moment of $u$ is

$$
\begin{aligned}
M_{u, r}=E u^{r} & =C(-1)^{r} \int_{0}^{\infty} u^{r} \exp \left(-\frac{1}{k} \frac{u^{k}}{(1-\lambda)^{k} \varphi^{k}}\right) d u \\
& +C \int_{0}^{\infty} u^{r} \exp \left(-\frac{1}{k} \frac{u^{k}}{(1+\lambda)^{k} \varphi^{k}}\right) d u
\end{aligned}
$$

Define

$$
t=\frac{1}{k} \frac{u^{k}}{(1 \pm \lambda)^{k} \varphi^{k}}
$$

and note that

$$
u=(1 \pm \lambda) \varphi k^{\frac{1}{k}} t^{\frac{1}{k}}
$$

and

$$
d u=(1 \pm \lambda) \varphi k^{\frac{1}{k}-1} t^{\frac{1}{k}-1} d t .
$$

Substitution of (A3) - (A5) into (A2) gives

$$
\begin{aligned}
M_{u, r}= & C(-1)^{r} k^{\frac{r+1}{k}-1}(1-\lambda)^{r+1} \varphi^{r+1} \int_{0}^{\infty} t^{\frac{r+1}{k}-1} e^{-t} d t \\
& +C k^{\frac{r+1}{k}-1}(1+\lambda)^{r+1} \varphi^{r+1} \int_{0}^{\infty} t^{\frac{r+1}{k}-1} e^{-t} d t
\end{aligned}
$$




$$
=C k^{\frac{r+1}{k}-1}\left[(-1)^{r}(1-\lambda)^{r+1}+(1+\lambda)^{r+1}\right] \varphi^{r+1} \Gamma\left(\frac{r+1}{k}\right)
$$

Setting $M_{u, 0}=1$, yields

$$
M_{u, 0}=C k^{\frac{1}{k}-1}[(1-\lambda)+(1+\lambda)] \varphi \Gamma\left(\frac{1}{k}\right)=2 C k^{\frac{1}{k}-1} \varphi \Gamma\left(\frac{1}{k}\right)=1,
$$

thus

$$
C=\frac{k^{1-\frac{1}{k}}}{2 \varphi} \Gamma\left(\frac{1}{k}\right)^{-1}
$$

Substitution of (A7) into (A1) and (A6) gives

$$
f(u)=\frac{k^{1-\frac{1}{k}}}{2 \varphi} \Gamma\left(\frac{1}{k}\right)^{-1} \exp \left(-\frac{1}{k} \frac{|u|^{k}}{(1+\operatorname{sgn}(u) \lambda)^{k} \varphi^{k}}\right)
$$

and

$$
M_{u, r}=\frac{1}{2}\left[(-1)^{r}(1-\lambda)^{r+1}+(1+\lambda)^{r+1}\right] G_{r} \varphi^{r}=A_{r} \varphi^{r}
$$

where

$$
G_{r}=k^{\frac{r}{k}} \Gamma\left(\frac{r+1}{k}\right) \Gamma\left(\frac{1}{k}\right)^{-1}
$$

and

$$
A_{r}=\frac{1}{2}\left[(-1)^{r}(1-\lambda)^{r+1}+(1+\lambda)^{r+1}\right] G_{r}
$$

The expected value of $u$ is

$$
E u=M_{u, 1}=A_{1} \varphi=2 \lambda G_{1} \varphi
$$

where

$$
G_{1}=k^{\frac{1}{k}} \Gamma\left(\frac{2}{k}\right) \Gamma\left(\frac{1}{k}\right)^{-1}
$$

The variance of $u$ 


$$
m_{2}=\operatorname{var}(u)=E u^{2}-(E u)^{2}=\left(A_{2}-A_{1}^{2}\right) \varphi^{2}
$$

where

$$
A_{2}=\frac{1}{2}\left[(-1)^{2}(1-\lambda)^{3}+(1+\lambda)^{3}\right] G_{2}=\left(1+3 \lambda^{2}\right) G_{2}
$$

and

$$
G_{2}=k^{\frac{2}{k}} \Gamma\left(\frac{3}{k}\right) \Gamma\left(\frac{1}{k}\right)^{-1}
$$

The third of $u$ is

$$
\begin{aligned}
M_{u, 3}=A_{3} \varphi^{3}= & \frac{1}{2}\left[(-1)^{3}(1-\lambda)^{4}+(1+\lambda)^{4}\right] G_{3} \varphi^{3} \\
& =2 \lambda\left(1+\lambda^{2}\right) G_{3} \varphi^{3}
\end{aligned}
$$

where

$$
G_{3}=k^{\frac{3}{k}} \Gamma\left(\frac{4}{k}\right) \Gamma\left(\frac{1}{k}\right)^{-1} .
$$

The fourth moment is

$$
\begin{aligned}
M_{u, 4}=A_{4} \varphi^{4} & =\frac{1}{2}\left[(-1)^{4}(1-\lambda)^{5}+(1+\lambda)^{5}\right] G_{4} \varphi^{4} \\
& =\left(1+10 \lambda^{2}+5 \lambda^{4}\right) G_{4} \varphi^{4}
\end{aligned}
$$

where

$$
G_{4}=k^{\frac{4}{k}} \Gamma\left(\frac{5}{k}\right) \Gamma\left(\frac{1}{k}\right)^{-1} .
$$

The third moment of $u$ is

$$
\begin{aligned}
m_{3}=E(u-E u)^{3} & =E u^{3}-3 E u^{2} E u+3 E u(E u)^{2}-(E u)^{3} \\
& =\left(A_{3}-3 A_{1} A_{2}+2 A_{1}^{3}\right) \varphi^{3}
\end{aligned}
$$

The fourth centered moment of $u$ is 


$$
\begin{gathered}
m_{4}=E(u-E u)^{4} \\
=E u^{4}-4 E u^{3} E u+6 E u^{2}(E u)^{2}-4 E u(E u)^{3}+(E u)^{4} \\
=\left(A_{4}-4 A_{3} A_{1}+6 A_{2} A_{1}^{2}-3 A_{1}^{4}\right) \varphi^{4} .
\end{gathered}
$$

The standardized skewness and kurtosis are

$$
S K=\frac{m_{3}}{m_{2}^{3 / 2}}=\frac{A_{3}-3 A_{1} A_{2}+2 A_{1}^{3}}{\left(A_{2}-A_{1}^{2}\right)^{3 / 2}}
$$

and

$$
K U=\frac{m_{4}}{m_{2}^{2}}=\frac{A_{4}-4 A_{3} A_{1}+6 A_{2} A_{1}^{2}-3 A_{1}^{4}}{\left(A_{2}-A_{1}^{2}\right)^{2}} .
$$

\section{Appendix B. Integral Evaluation Under Normality}

Under normality, the integral

$$
\begin{gathered}
\int_{h}^{\infty} e^{z \sigma_{a} \sqrt{\tau}} f(z) d z=\int_{h}^{\infty} e^{z \sigma_{a} \sqrt{\tau}} \frac{1}{\sqrt{2 \pi}} e^{-\frac{1}{2} z^{2}} d z \\
=e^{\frac{1}{2} \sigma_{a}^{2} \tau} \int_{\infty}^{-h} \frac{1}{\sqrt{2 \pi}} e^{-\frac{1}{2}\left(z-\sigma_{a} \sqrt{\tau}\right)^{2}} d z \\
=e^{\frac{1}{2} \sigma_{a}^{2} \tau} \int_{\infty}^{-h+\sigma_{a} \sqrt{\tau}} \frac{1}{\sqrt{2 \pi}} e^{-\frac{1}{2} s^{2}} d s=e^{\frac{1}{2} \sigma_{a}^{2} \tau} \Phi\left(-h+\sigma_{a} \sqrt{\tau}\right)
\end{gathered}
$$

where $s=z-\sigma_{a} \sqrt{\tau}, d z=d s$ and $\Phi$ is the cumulative density for the standard normal distribution. 


\section{References}

Akgiray, V. 1989. "Conditional Heteroscedasticity in Time Series of Stock Returns: Evidence and Forecasts." Journal of Business 62: 55-80.

Baillie, R. T., and Bollerslev, T. 1989. "The Message in Daily Exchange Rates: A Conditional Variance Tale." Journal of Business and Economic Statistics 7: 297-305.

Biger, N., and Hull, J. 1983. "The Valuation of Currency Options." Financial Management 12: 24-28.

Black, F. 1976. "The Pricing of Commodity Contracts." Journal of Financial Economics 3: 167-179.

Black, F., and Scholes, M. 1973. "The Pricing of Options and Corporate Liabilities." Journal of Political Economy 81: 637-59.

Bookstaber, R. M., and McDonald, J. B. 1987. "A General Distribution for Describing Security Price Returns." Journal of Business 60: 401-424.

Bollerslev, T. 1987. "A Conditional Heteroskedastic Time Series Model for Speculative Prices and Rates of Return." Review of Economics and Statistics 69: 542-547.

Booth, G. G.; Martikainen, T.; and Tse, Y. 1997. "Price and Volatility Spillovers in Scandinavian Stock Markets." Journal of Banking and Finance, 21. Reprinted in Volatility: New Estimation Techniques for Pricing Derivatives, ch.19, section IV, 1998. Ed: R. Jarrow. London: Risk Books.

Box, G. E. P., and Tiao, G. C. 1962. "A Further Look at Robustness Via Bayes's Theorem." Biometrika 49: 419-432.

Butler, R. J.; McDonald, J. B.; Nelson, R. D.; and White, S. B. 1990. "Robust and Partially Adaptive Estimation of Regression Models." Review of Economics and Statistics: 321-327.

Chiang, T.; Tan, L.; Li, J.; and Nelling, E. 2013. "Dynamic Herding Behavior in Pacific-Basin Markets: Evidence and Implications." Multinational Finance Journal 17: 165-200.

Délèze, F., and Hussain S. M. 2014. "Information Arrival, Jumps and Cojumps in European Financial Markets: Evidence Using Tick by Tick Data." Multinational Finance Journal 18: 169-213.

Engle, R. F.; and Gonzalez-Rivera, G. 1991. "Semiparametric ARCH Models." Journal of Business and Economics Statistics 9: 345-359.

Ferguson, N.J.; Philip, D.; Lam H.Y.; and Guo, J.M. 2015. "Media Content and Stock Returns: The Predictive Power of Press." Multinational Finance Journal 19: 1-31.

Gulati, A. B.; Kolari, J. W.; and Knif, J. 2013. "Exchange Rate Shocks and Firm Competitiveness in a Small, Export-Oriented Economy: The Case of Finland." Multinational Finance Journal 17: 1-47.

Hsieh, D. 1989. "Modeling Heteroskedasticity in Daily Foreign Exchange Rates." Journal of Business and Economics Statistics 7: 307-317. 
Jarrow, R., and Rudd, A. 1982. "Approximate Option Valuation for Arbitrary Stochastic Processes." Journal of Financial Economics 10: 347-369.

Johnson, N. L.; Kotz, S.; and Balakrishnan, N. 1995. Continuous Univariate Distributions, vol. 2, 2nd edition. New York: John Wiley.

McDonald, J. B., and Bookstaber, R. M. 1991. "Option Pricing for Generalized Distributions." Communications in Statistics: Theory and Methods 20(12): 4053-4068.

McDonald, J. B., and Newey, W. K. 1988. "Partially Adaptive Estimation of Regression Models Via the Generalized t Distribution." Econometric Theory 4, 1988: 428-457.

McDonald, J. B., and Nelson, R. D. 1989. "Alternative Beta Estimation for the Market Model Using Partially Adaptive Techniques." Communications in Statistics: Theory and Methods 18: 4039-4058.

Merton, R. C. 1973. "Theory of Rational Option Pricing." Bell Journal of Economics and Management Science 4: 141-183.

Neftci, S. N. 1996. An Introduction to the Mathematics of Financial Derivatives. San Diego: Academic Press.

Nelson, D. 1991. "Conditional Heteroskedasticity in Asset Returns: A New Approach." Econometrica 59: 347-370.

Ross, S. M. 1999. An Introduction to Mathematical Finance: Options and Other Topics. New York: Cambridge University Press.

Stuart, A., and Ord, K. 1994. Kendall's Advanced Theory of Statistics: Distribution Theory, vol. 1, 6th Edition. London: Edward Arnold.

Subbotin, M. T. 1923. "On the Law of Frequency of Error." Matematicheskii Sbornik 31: 296-301.

Tapia, R. A., and Thompson, J. R. 1978. Nonparametric Probability Density Estimation. Baltimore: Johns Hopkins University Press.

Theodossiou, P. 1998. "Financial Data and the Skewed Generalized t Distribution." Management Science 44 (12-1): 1650-1661.

Theodossiou, P., and Savva, C. 2016. "Skewness and the Relation Between Risk and Return.” Management Science, Forthcoming. 